\title{
LARGE DEVIATIONS FOR BROWNIAN MOTION IN A RANDOM POTENTIAL
}

\author{
DAniel Boivin* AND Thi Thu Hien Lê
}

\begin{abstract}
A quenched large deviation principle for Brownian motion in a stationary potential is proved. As the proofs are based on a method developed by Sznitman [Comm. Pure Appl. Math. 47 (1994) 1655-1688] for Brownian motion among obstacles with compact support no regularity conditions on the potential is needed. In particular, the sufficient conditions are verified by potentials with polynomially decaying correlations such as the classical potentials studied by Pastur [Teoret. Mat. Fiz. 32 (1977) 88-95] and Fukushima [J. Stat. Phys. 133 (2008) 639-657] and the potentials recently introduced by Lacoin [Ann. Inst. Henri Poincaré Probab. Stat. 48 (2012) 1010-1028; 1029-1048].
\end{abstract}

Mathematics Subject Classification. 82B41, 60K37.

Received December 3, 2019. Accepted February 4, 2020.

\section{INTRODUCTION AND THE MAIN RESULT}

Consider a standard Brownian motion on $\mathbb{R}^{d},\left(Z_{s} ; s \geq 0\right)$, moving in a non-negative stationary ergodic potential. That is, it is assumed that the potential is of the form

$$
V(x, \omega):=V_{0}\left(\tau_{x} \omega\right), \quad x \in \mathbb{R}^{d}, \omega \in \Omega
$$

where $V_{0}$ is a real-valued non-negative random variable not identically zero on a probability space $(\Omega, \mathcal{F}, \mathbb{P})$ and $\left(\tau_{x} ; x \in \mathbb{R}^{d}\right)$ is a family of measurable maps on $(\Omega, \mathcal{F}, \mathbb{P})$ which satisfies

$$
\begin{aligned}
& \tau_{x} \circ \tau_{y}=\tau_{x+y} \text { for all } x, y \in \mathbb{R}^{d}, \\
& (x, \omega) \mapsto \tau_{x} \omega \text { is measurable on the cartesian product } \mathbb{R}^{d} \times \Omega, \\
& \mathbb{P} \text { is invariant under } \tau_{x} \text { for all } x \in \mathbb{R}^{d} \text { and } \\
& \text { is ergodic, that is, if for some } A \in \mathcal{F}, \tau_{x}(A)=A \text { for all } x \in \mathbb{R}^{d} \text { then } \mathbb{P}(A)=0 \text { or } 1 \text {. }
\end{aligned}
$$

The quenched path measures are defined by

$$
Q_{t, \omega}:=\frac{1}{S_{t, \omega}} \exp \left(-\int_{0}^{t} V\left(Z_{s}, \omega\right) \mathrm{d} s\right) P_{0}, \quad t>0, \omega \in \Omega
$$

Keywords and phrases: Brownian motion, long-range random potential, Lyapunov exponents, shape theorem, large deviations. Univ Brest, Université de Brest, LMBA UMR CNRS 6205, 6 avenue Le Gorgeu, 29238 Brest cedex, France.

* Corresponding author: daniel.boivin@univ-brest.fr 
where the normalizing constants $S_{t, \omega}$ are the quenched survival functions up to time $t$

$$
S_{t, \omega}:=E_{0}\left[\exp \left(-\int_{0}^{t} V\left(Z_{s}, \omega\right) \mathrm{d} s\right)\right], \quad t>0, \omega \in \Omega
$$

Here $P_{x}$ is the Wiener measure on paths starting from $x \in \mathbb{R}^{d}$ and $E_{x}$ is the expectation with respect to $P_{x}$.

In ([36], Thm. 0.1) (see also [37], Sect. 5.4), Sznitman proved a quenched large deviation principle for the speed of the Brownian motion in a Poissonian potential constructed from obstacles with compact support. Building on this work, Armstrong and Tran ([1], Cor. 2) proved a quenched LDP for a wide class of Hamiltonians with stationary potentials. However, the homogenization techniques used in [1] require some regularity of the potential. In particular, the sufficient condition given for the LDP involves a finite moment of the Lipschitz norm of the potential. The goal of this paper is to extend the quenched LDP for the speed of the Brownian motion in a random potential to stationary random potentials without imposing a regularity condition.

Our LDP is stated below in Theorem 1.1. Besides stationarity and ergodicity, the sufficient conditions in Theorem 1.1 involve an integrability condition expressed in terms of the Lorentz spaces and the principal eigenvalue of $-\frac{1}{2} \Delta+V$. We first recall these two notions and then we state Theorem 1.1.

The Lorentz spaces (see for instance [2, p. 634], or [4]) which appear in our context are defined as

$$
L_{\mathbb{P}}(d, 1)=\left\{f:(\Omega, \mathcal{F}) \rightarrow(\mathbb{R}, \mathcal{B}(\mathbb{R})) \text { is measurable and }\|f\|_{d, 1}<\infty\right\}
$$

where $\|f\|_{d, 1}=\int_{0}^{1} f^{*}(s) s^{(1 / d)-1} \mathrm{~d} s$ and $f^{*}:[0,1] \rightarrow \mathbb{R}^{+}$is the non-increasing right continuous function which has the same distribution as $|f|$. Note that $L_{\mathbb{P}}(d, 1)$ is a Banach space and there are positive constants $c_{1}$ and $c_{2}$ such that for all $\varepsilon>0$

$$
c_{1}\|f\|_{d} \leq\|f\|_{d, 1} \leq c_{2}\|f\|_{d+\varepsilon} \quad \text { where }\|f\|_{p}^{p}=\int_{\Omega}|f|^{p} d \mathbb{P} .
$$

In particular, $L_{\mathbb{P}}^{d+\varepsilon} \subset L_{\mathbb{P}}(d, 1) \subset L_{\mathbb{P}}^{d}$ for all $\varepsilon>0$.

The principal Dirichlet eigenvalue of $-\frac{1}{2} \Delta+V$ is defined as

$$
\lambda_{V}:=\inf \left\{\int_{\mathbb{R}^{d}}\left(\frac{1}{2}|\nabla \varphi|^{2}+V \varphi^{2}\right) \mathrm{d} x ; \varphi \in \mathcal{C}_{c}^{\infty}\left(\mathbb{R}^{d}\right), \int_{\mathbb{R}^{d}} \varphi^{2} \mathrm{~d} x=1\right\} .
$$

By ergodicity, $\lambda_{V}$ is non-random. It is closely related to the asymptotic behavior of the survival function. Indeed,

$$
\lim _{t \rightarrow \infty}-\frac{1}{t} \log S_{t, \omega}=\lambda_{V} \quad \mathbb{P}-\text { a.s. }
$$

A proof is given in ([37], Sect. 3.1) for non-negative potentials in the Kato class $\mathcal{K}_{d}^{\text {loc }}$. These include the stationary potentials which satisfy conditions (1.6) and (1.8) below.

Denote the Lebesgue measure on $\mathbb{R}^{d}$ by Leb and the expectation with respect to $\mathbb{P}$ by $\mathbb{E}$. The Euclidean ball $\left\{x \in \mathbb{R}^{d} ;|x-y|<R\right\}$ will be denoted by $B(y, R)$ and $B(y):=B(y, 1)$.

Theorem 1.1. Let $V$ be a non-negative, stationary and ergodic potential which satisfies

$$
\sup _{x \in B(0)} V(x, \cdot) \in L_{\mathbb{P}}(d, 1) .
$$

and

$$
\lambda_{V}=\inf _{\Omega} V_{0}
$$


For $d=1$ or 2, suppose moreover that there exist positive constants $\rho, \varepsilon$ and a measurable function $u: \Omega \rightarrow \mathbb{R}^{d}$ such that $\mathbb{P}$ - a.s.

$$
\operatorname{Leb}\left(\left\{x \in \mathbb{R}^{d} ; V(x, \omega)>\varepsilon\right\} \cap B(u(\omega), \rho)\right)>\varepsilon \text { and } \mathbb{E}\left(|u(\cdot)|^{d}\right)<\infty .
$$

Then there is a deterministic, continuous convex rate function $I: \mathbb{R}^{d} \rightarrow[0, \infty[$ given in (2.10), with level sets $\left\{x \in \mathbb{R}^{d} ; I(x) \leq c\right\}$ that are compact for all $c \in \mathbb{R}$ and such that, $\mathbb{P}$ - a.s.,

for all closed subsets $A$ of $\mathbb{R}^{d}$,

$$
\limsup _{t \rightarrow \infty} \frac{1}{t} \log Q_{t, \omega}\left(Z_{t} \in t A\right) \leq-\inf _{x \in A} I(x)
$$

and for all open subsets $\mathcal{O}$ of $\mathbb{R}^{d}$,

$$
\liminf _{t \rightarrow \infty} \frac{1}{t} \log Q_{t, \omega}\left(Z_{t} \in t \mathcal{O}\right) \geq-\inf _{x \in \mathcal{O}} I(x) .
$$

The expression of the rate function in terms of Lyapunov exponents allows to prove that the change in regime of the Brownian motion with constant drift observed by Sznitman ([36], Thm. 0.3) in a Poissonian potential associated to obstacles with compact support actually occurs for a large class of measurable potentials. This phase transition was further studied by Flury $[14,15]$ both in the discrete and the continuous settings. Concurrently, also under some regularity conditions on the potential, Ruess [33] proved the existence of the Lyapunov exponents for Brownian motion in stationary potentials. It does not seem possible to extend his results by approximating a measurable potential by regular potentials. Especially since Ruess [34] gave an example where the Lyapunov exponents are not continuous with respect to the potential.

For random walks in a random potentials there is an extensive literature starting with Varadhan [38] who proved both a quenched and an annealed LDP for the speed of a uniformly elliptic random walk. In his thesis, Rosenbluth [32] proved a quenched large deviation principle for a large class of random walks on $\mathbb{Z}^{d}$ with stationary transition probabilities under an integrability condition similar to condition (2.2). In these works, the quenched rate function is expressed as a variational formula in terms of cocycles.

Intensive work to extend both the class of random walks and the class of potentials for which a LDP holds was undertaken by Rassoul-Agha, Seppäläinen, Yilmaz. Some of these results, which include level-3 LDP can be found in [28-31, 42, 43]. Yilmaz and Zeitouni [44] studied a class of random walks in a random environment where the annealed and quenched rate functions differ.

Sznitman's method, based on Lyapunov exponents, was also used to obtain a LDP for random walks in a random potential in $[14,45]$. Mourrat [25] considered the simple random walk in an i.i.d. potential taking values in $[0 ;+\infty]$ and showed a LDP without assuming a moment condition on $V$. See also [23].

In this paper, we also follow Sznitman's method. Along the way, we provide sufficient conditions for the intermediate results. The existence of the Lyapunov exponents is shown in Theorem 2.1 for stationary potentials under a weaker integrability condition than (1.6). Then under (1.6), we prove in the shape Theorem 2.2 that the convergence is uniform with respect to the direction. The appropriate tool in this context is provided by Björklund's generalization of the shape Theorem [2].

The main difficulty is in the proof of (1.10) under the additional condition on the principal Dirichlet eigenvalue. We will show how key arguments of ([36], Sect. 2) can be done on a linear scale. This will permit the use of the maximal inequality for cocycles ([3], Cor. 2) and of a technique introduced in [4]. See also [5] for an application in a different context.

The results are stated in Section 2 and the proofs are given in Section 3.

In the last section, we will verify the sufficient conditions for the LDP for long-range Poissonian potentials. These were considered in a previous version of this paper. They are of the form

$$
V(x, \omega)=\sum_{j} W\left(x-\omega_{j}\right), \quad x \in \mathbb{R}^{d},
$$


where $\omega=\left(\omega_{j} ; j \in \mathbb{N}\right)$ is a Poisson cloud in $\mathbb{R}^{d}, d \geq 1$ and $W(x)=|x|^{-\gamma} \wedge 1$ with $\gamma>d$. Moreover, for these potentials, it is possible to show that $I(x)>0$ for $x \in \mathbb{R}^{d} \backslash\{0\}$.

Potentials constructed in Section 4.1 from a Boolean model also satisfy the sufficient conditions of the LDP. We end the last section with the presentation a model introduced by Ruess [34] which does not have decorrelation properties but still satisfies a large deviation principle.

Notations. For $y \in \mathbb{R}^{d}$ and $R>0$, the Euclidean norm of $y$ is denoted by $|y|$ and $B(y, R)$ is the Euclidean ball $\left\{x \in \mathbb{R}^{d} ;|x-y|<R\right\} . B(y)$ stands for the unit ball $B(y, 1)$ and $H(y)=\inf \left\{t \geq 0: Z_{t} \in \bar{B}(y)\right\}$ is the hitting time of $\bar{B}(y)$, the closure of $B(y)$. For an open set $D \subset \mathbb{R}^{d}, T_{D}:=\inf \left\{t \geq 0, Z_{t} \notin D\right\}$ and $\mathcal{C}_{c}^{\infty}(D)$ is the space of infinitely differentiable functions with compact support in $D$. For $x \in \mathbb{R}^{d},[x]$ is the element of $\mathbb{Z}^{d}$ closest to $x$, with some fixed rule for ties.

The Lebesgue measure on $\mathbb{R}^{d}$ is denoted by Leb and the volume of the unit ball of $\mathbb{R}^{d}$ by $\mathcal{L}_{d}$. The principal Dirichlet eigenvalue of $-\frac{1}{2} \Delta$ in the unit ball is denoted by $\lambda_{d}$.

For a random variable $X$ and for $A \in \mathcal{F}$, let $E[X, A]:=E\left(X \mathbf{1}_{A}\right)$.

The constants, whose value may vary from line to line, are denoted by $c$ or $C$. Some are numbered for subsequent reference.

\section{OVERVIEW OF THE RESULtS}

In this section, the existence of Lyapunov exponents of a Brownian motion in a stationary potential and the shape theorem will be proved under appropriate moment conditions. Then we will show how Sznitman's method leads to a large deviation principle.

Recently, Ruess [33] considered Brownian motion in a stationary potential. Inspired by Schröder [35], he showed the existence of Lyapunov exponents for a large class of potentials and he expressed them in terms of a variational formula. However, the existence of Lyapunov exponents by itself follows from the subadditive theorem under much weaker assumptions on the potential.

For $x, y \in \mathbb{R}^{d}$ and $\omega \in \Omega$, define

$$
\begin{aligned}
& e(x, y, \omega):=E_{x}\left[\exp \left(-\int_{0}^{H(y)} V\left(Z_{s}, \omega\right) \mathrm{d} s\right), H(y)<\infty\right] \\
& a(x, y, \omega):=-\log e(x, y, \omega) .
\end{aligned}
$$

The measurability of $e(x, y, \omega)$ can be verified by standard arguments. It rests on the hypothesis that $(x, \omega) \mapsto$ $\tau_{x} \omega$ is measurable on $\mathbb{R}^{d} \times \Omega$. Moreover, under the condition $\mathbb{E}\left[\sup _{x \in B(0)} V(x, \cdot)\right]<\infty$, the potential $V$ locally belongs to the Kato class $\mathcal{K}_{d}^{\text {loc }}$ and the probabilities $e(x, y, \omega)$ are strictly positive (cf. [37], Sects. 1.2 and 5.2).

We introduce the Green measure relative to the potential $V$ :

$$
G(x, A, \omega):=E_{x}\left[\int_{0}^{\infty} \mathbf{1}_{A}\left(Z_{t}\right) \exp \left(-\int_{0}^{t} V\left(Z_{s}, \omega\right) \mathrm{d} s\right) \mathrm{d} t\right]
$$

where $x \in \mathbb{R}^{d}, \omega \in \Omega$ and $A$ is a Borel subset of $\mathbb{R}^{d}$. $G$ can be interpreted as the expected occupation time measure of Brownian motion killed at rate $V(\cdot, \omega)$. We define $g(x, y, \omega)$ as the density function relative to the Green measure and we call it the Green function. The existence of $g$ is proved in ([37], (2.2.3)).

We show in the next theorem that the Green function as well as the probabilities $e(x, y, \omega)$ have exponential decay rates which are called Lyapunov exponents. Theorem 2.2 shows that, under a stronger moment condition, the convergence to the Lyapunov exponents is uniform with respect to the directions.

Theorem 2.1 (Existence of Lyapunov exponents). Let $V$ be a non-negative, stationary and ergodic potential which satisfies

$$
\mathbb{E}\left[\sup _{x \in B(0)} V(x, \cdot)\right]<\infty
$$


For $d=1,2$, assume moreover that (1.8) holds.

Then there is a non-random semi-norm $\alpha(\cdot)$ on $\mathbb{R}^{d}$ such that $\mathbb{P}$-a.s. and in $L_{\mathbb{P}}^{1}$, for all $x \in \mathbb{R}^{d}$

$$
\lim _{r \rightarrow \infty} \frac{1}{r} a(0, r x, \omega)=\lim _{r \rightarrow \infty} \frac{1}{r} \mathbb{E}[a(0, r x, \omega)]=\inf _{r>0} \frac{1}{r} \mathbb{E}[a(0, r x, \omega)]=\alpha(x) .
$$

$\alpha$ is called the quenched Lyapunov exponent.

$a(0, x, \omega)$ can be replaced by $-\log g(0, x, \omega)$ in (2.3).

Björklund [2] extended to a very general context the shape theorem proved in [10] for first-passage percolation with independent passage times and in [4] for stationary passage times. This theorem can be applied in our framework.

Theorem 2.2 (Shape theorem). Let $V$ be a non-negative, stationary and ergodic potential which satisfies (1.6) and (1.8).

Then $\mathbb{P}$ - a.s., as $x \rightarrow \infty, x \in \mathbb{R}^{d}$,

$$
\frac{1}{|x|}|a(0, x, \omega)-\alpha(x)| \rightarrow 0
$$

$a(0, x, \omega)$ can be replaced by $-\log g(0, x, \omega)$ in (2.4).

For the Proof of Theorems 2.1 and 2.2, we need to define

$$
d(x, y, \omega):=\max \left(-\inf _{B(x)} \log e(\cdot, y, \omega),-\inf _{B(y)} \log e(x, \cdot, \omega)\right), \quad x, y \in \mathbb{R}^{d}, \omega \in \Omega .
$$

By using the strong Markov property of Brownian motion, it is simple to verifiy that $d(\cdot, \cdot, \omega)$ is a semi-norm on $\mathbb{R}^{d}$. By ([37], Lem. 5.2.1), $\mathbb{P}$ - a.s. $d(\cdot, \cdot, \omega)$ defines a distance on $\mathbb{R}^{d}$ which induces the usual topology. These properties still hold in a stationary potential.

Theorem 2.2 is first proved for the distance $d(0, x, \omega)$. Then Lemmas 2.3 and 2.4 allows to replace $d(0, x, \omega)$ by $a(0, x, \omega)$ or by $-\log g(0, x, \omega)$ in equation $(2.4)$.

Define

$$
F_{0}(\omega):=\log ^{+}\left(\int_{B(0) \times B(0)} g(x, y, \omega) \mathrm{d} x \mathrm{~d} y\right)+\sup _{\bar{B}(0)} V(\cdot, \omega), \quad \omega \in \Omega
$$

and let $F(x, \omega):=F_{0}\left(\tau_{x} \omega\right)$.

The proof of the following lemma can be found in ([37], Prop. 5.2.2). The proof is very general as it requires only basic notions of potential theory.

Lemma 2.3. Let $V$ be a non-negative, stationary and ergodic potential which satisfies condition (2.2) and (1.8).

Then there exists a positive constant $C$ such that for $x, y \in \mathbb{R}^{d},|x-y|>4, \mathbb{P}-$ a.s.

$$
\max (|d(x, y, \omega)+\log g(x, y, \omega)|,|d(x, y, \omega)-a(x, y, \omega)|) \leq C(1+F(x, \omega)+F(y, \omega)) .
$$

Lemma 2.4. Let $V$ be a non-negative, stationary and ergodic potential.

(i) If (2.2), and (1.8) when $d=1$ or 2 , hold, then for all $x \in \mathbb{Z}^{d}, \mathbb{P}$ - a.s.,

$$
\lim _{k \rightarrow \infty} \frac{F(k x, \omega)}{k}=\lim _{k \rightarrow \infty} \mathbb{E} \frac{F(k x, \omega)}{k}=0 .
$$

(ii) If (1.6), and (1.8) when $d=1$ or 2 , hold, then $\mathbb{P}$ - a.s.,

$$
\lim _{x \rightarrow \infty, x \in \mathbb{Z}^{d}} \frac{F(x, \omega)}{|x|}=0 .
$$


The rate function of large deviation principle will be given in terms of the Lyapunov exponents $\alpha_{\lambda}(x)$ associated with the potential $\lambda+V$ where $\lambda \geq-\underline{V}$ where $\underline{V}:=\inf _{\Omega} V$. The essential properties of $\alpha_{\lambda}$ are gathered in the next lemma. The upper bound (2.8) should be compared with ([37], (5.2.31)) and with ([45], (65)) for a random walk in a random potential.

Note that, as in [45, section 6], the results will be stated in terms of $\underline{V}$ as it highlights the role of the principal eigenvalue $\lambda_{V}$ and it facilitates the comparison with the results from stochastic homogenization.

Lemma 2.5. Let $V$ be a non-negative, stationary and ergodic potential which satisfies (1.6) and (1.8).

Then $(\lambda, x) \mapsto \alpha_{\lambda}(x)$ is a continuous function on $\left[-\underline{V}, \infty\left[\times \mathbb{R}^{d}\right.\right.$, for $x \in \mathbb{R}^{d}, \lambda \mapsto \alpha_{\lambda}(x)$ is a concave increasing function on $\left[-\underline{V}, \infty\left[\right.\right.$, for all $x \in \mathbb{R}^{d}$ and $\lambda \geq-\underline{V}$,

$$
\sqrt{2(\lambda+\underline{V})}|x| \leq \alpha_{\lambda}(x) \leq|x| \sqrt{2\left(\lambda+\lambda_{d}+\mathbb{E} \sup _{B(0)} V\right)}
$$

and for all $x \neq 0$,

$$
\alpha_{\lambda}^{\prime}(x)_{-} \rightarrow 0 \text { as } \lambda \rightarrow \infty
$$

In Proposition 2.9 of [37], the lower bound for the Lyapunov exponents has the form

$$
\alpha_{\lambda}(x) \geq \max (\sqrt{2(\lambda+\underline{V})}, C)|x|, \quad x \in \mathbb{R}^{d}
$$

for some positive constant $C$. In particular this implies the non-degeneracy of $\alpha_{0}(\cdot)$. But the proof requires specific properties of Poissonian potentials. This lower bound is proved for some long-range potentials in ([23], $(2.87))$.

The rate function of the LDP will be given by

$$
I(x):=\sup _{\lambda \geq-\underline{V}}\left(\alpha_{\lambda}(x)-\lambda\right)-\underline{V}, \quad x \in \mathbb{R}^{d} .
$$

Bounds on the rate function are easily obtained from the estimates on the Lyapunov exponents given in (2.8). When combined with the convexity properties of the Lyapunov exponents, we obtain the following properties of the rate function. See also ([37], Lem. 5.4.1).

Lemma 2.6. Let $V$ be a non-negative, stationary and ergodic potential which satisfies (1.6) and (1.8).

Then for all $x \in \mathbb{R}^{d}$,

$$
\frac{|x|^{2}}{2} \leq I(x) \leq \frac{|x|^{2}}{2}+\lambda_{d}+\mathbb{E} \sup _{B(0)} V
$$

and $I: \mathbb{R}^{d} \rightarrow\left[0, \infty\left[\right.\right.$ is a non-negative convex continuous function such that the sets $\left\{x \in \mathbb{R}^{d} ; I(x) \leq c\right\}$ are compact for all $c \in \mathbb{R}$.

Armstrong and Tran [1] obtained a large deviation principle for a diffusion in a stationary convex Hamiltonian with some regularity and under a weak coercivity condition. In the particular case of a Brownian motion in a random potential, the Hamiltonian is given by

$$
H(p, y):=\frac{1}{2} p^{2}-V(y, \omega), \quad p, y \in \mathbb{R}^{d} .
$$

Although it does not appear explicitly in [37], a central object in stochastic homogenization is the effective Hamiltonian $\bar{H}$ which appears in the homogenized problem. It is a non-random, continuous and convex function 
from $\mathbb{R}^{d}$ to $\mathbb{R}$. It also satisfies, see ([1], Sect. 6$)$,

$$
\bar{H}(0)=\min _{p \in \mathbb{R}^{d}} \bar{H}(p)=-\lambda_{V}
$$

The rate function of the LDP principle is given in ([1], Cor. 2) by,

$$
I_{A T}(x):=\bar{L}(x)+\bar{H}(0), \quad x \in \mathbb{R}^{d},
$$

where $\bar{L}$ is Legendre-Fenchel transform of $\bar{H}$, that is $\bar{L}(x):=\sup _{p \in \mathbb{R}^{d}}(p \cdot x-\bar{H}(p))$.

Note that the estimates on $\bar{H}(p)$ given in ([1], Lem. 3.1) lead to estimates on the rate function. Therefore, as in Lemma 2.6, the rate function of a wide class of Hamiltonians is a non-random convex and continuous function with compact level sets.

In $([1],(3.2))$, the non-random functions $\bar{m}_{\mu}(x)$, which are analogous to the Lyapunov exponents, are also expressed in terms of $\bar{H}$ as $\bar{m}_{\mu}(x)=\sup _{p}\{p \cdot x ; \bar{H}(p) \leq \mu\}$ with the convention that $\sup \emptyset=-\infty$.

Then to see that, under the condition (1.7), the rate function $I_{A T}$ coincide with the rate function given in (2.10), one can proceed as in ([1], Sect. 1.3): For $x \in \mathbb{R}^{d}$, by (2.12),

$$
\begin{aligned}
\bar{L}(x) & :=\sup _{p \in \mathbb{R}^{d}}(p \cdot x-\bar{H}(p)) \\
& =\sup _{\mu \geq \bar{H}(0)} \sup _{p \in \mathbb{R}^{d}}\{p \cdot x-\bar{H}(p) ; \bar{H}(p) \leq \mu\} \\
& =\sup _{\mu \geq \bar{H}(0)} \sup _{p \in \mathbb{R}^{d}}\{p \cdot x-\mu ; \bar{H}(p) \leq \mu\} \\
& =\sup _{\mu \geq \bar{H}(0)}\left\{\bar{m}_{\mu}(x)-\mu\right\} .
\end{aligned}
$$

With a "gauge theorem" ([9], Chap. 4), one could also give an analogue of $\bar{H}_{*}$ by describing $\lambda_{V}$ in terms of the existence of a solution of $\frac{\Delta}{2} u-V u+\lambda u=0$ in the appropriate Sobolev space for an increasing sequence of domains.

\section{PROOFS}

Proof of Lemma 2.4. For $d \geq 3$, there is a positive constant $C$ such that $\mathbb{P}$ a.s. for all $x \neq y, g(x, y, \omega) \leq$ $C|x-y|^{2-d}$. Hence

$$
\int_{B(0) \times B(0)} g(x, y, \omega) \mathrm{d} x \mathrm{~d} y \leq \int_{B(0) \times B(0)} C|x-y|^{2-d} \mathrm{~d} x \mathrm{~d} y<\infty .
$$

Fix $x \in \mathbb{R}^{d}$. Put $X_{k}(\omega):=\sup _{\bar{B}(k x)} V(\cdot, \omega)$. Then by the stationarity of the potential and by condition $(2.2)$, $\left(X_{k} ; k \geq 0\right)$ is a stationary sequence of non-negative random variables with finite expectation. Then by BorelCantelli lemma, $\mathbb{P}$ - a.s., $\lim _{k \rightarrow \infty} \frac{X_{k}}{k}=0$. It follows that condition (2.6) is satisfied for $d \geq 3$.

For $d=1$ or 2 , assume that condition (1.8) is satisfied for some positive numbers $\rho, \varepsilon$ and for $u: \Omega \rightarrow \mathbb{R}^{d}$ such that $\mathbb{E}|u|<\infty$.

Consider $D:=B(0,|u|+2 \rho+1)$. Construct two increasing sequences of stopping times with respect to the natural right continuous filtration $\left(\mathcal{F}_{t}\right)$ on $\mathcal{C}\left(\mathbb{R}^{+}, \mathbb{R}^{d}\right)$. These stopping times describe the successive times of return to $\bar{B}(0)$ and exit times from $D$ of the Brownian motion

$$
R_{1}:=\inf \left\{t \geq 0: Z_{t} \in \bar{B}(0)\right\}, \quad T_{1}:=\inf \left\{t \geq R_{1}, Z_{t} \notin D\right\}
$$

and by induction for $n \geq 1, R_{n+1}=R_{1} \circ \theta_{T_{n}}+T_{n}, T_{n+1}=T_{1} \circ \theta_{R_{n}}+R_{n}$ where $\theta_{t}, t \geq 0$ is the canonical shift on $\mathcal{C}\left(\mathbb{R}^{+}, \mathbb{R}^{d}\right)$. 
Since the Brownian motion is recurrent when $d=1$ or 2 , the stopping times are a.s. finite and

$$
0 \leq R_{1}<T_{1}<R_{2}<T_{2}<\cdots<R_{n}<T_{n} \cdots \text { and } R_{n}, T_{n} \uparrow \infty .
$$

We now have for $x \in \mathbb{R}^{d}$,

$$
\begin{aligned}
\int_{\bar{B}(0)} g(x, y, \omega) \mathrm{d} y & =E_{x}\left[\int_{0}^{\infty} \mathbf{1}_{\bar{B}(0)}\left(Z_{t}\right) \exp \left(-\int_{0}^{t} V\left(Z_{s}\right) \mathrm{d} s\right) \mathrm{d} t\right] \\
& =E_{x}\left[\sum_{i \geq 1} \int_{R_{i}}^{T_{i}} \mathbf{1}_{\bar{B}(0)}\left(Z_{t}\right) \exp \left(-\int_{0}^{t} V\left(Z_{s}\right) \mathrm{d} s\right) \mathrm{d} t\right] \\
& \leq \sum_{i=1}^{\infty} E_{x}\left[\exp \left(-\int_{0}^{R_{i}} V\left(Z_{s}\right) \mathrm{d} s\right) \int_{R_{i}}^{T_{i}} \mathbf{1}_{\bar{B}(0)}\left(Z_{t}\right) \mathrm{d} t\right] \\
& =\sum_{i=1}^{\infty} E_{x}\left[\exp \left(-\int_{0}^{R_{i}} V\left(Z_{s}\right) \mathrm{d} s\right) E_{Z_{R_{i}}}\left[\int_{0}^{T_{1}} \mathbf{1}_{\bar{B}(0)}\left(Z_{t}\right) \mathrm{d} t\right]\right]
\end{aligned}
$$

by the strong Markov property,

$$
\begin{aligned}
& \leq \sup _{x \in \bar{B}(0)} E_{x}\left(T_{D}\right) \sum_{i=1}^{\infty} E_{x}\left[\exp \left(-\int_{0}^{R_{i}} V\left(Z_{s}\right) \mathrm{d} s\right)\right] \\
& \leq C(|u|+2 \rho+1)^{2} \sum_{i=1}^{\infty} E_{x}\left[\exp \left(-\int_{0}^{R_{i}} V\left(Z_{s}\right) \mathrm{d} s\right)\right] .
\end{aligned}
$$

Now, for $i \geq 1$, by the strong Markov property and by induction, for all $x \in \bar{B}(0)$,

$$
\begin{aligned}
E_{x}\left[\exp \left(-\int_{0}^{R_{i+1}} V\left(Z_{s}\right) \mathrm{d} s\right)\right] & \leq E_{x}\left[\exp \left(-\int_{0}^{R_{i}} V\left(Z_{s}\right) \mathrm{d} s\right) E_{Z_{R_{i}}}\left[\exp \left(-\int_{0}^{T_{D}} V\left(Z_{s}\right) \mathrm{d} s\right)\right]\right] \\
& \leq E_{x}\left[\exp \left(-\int_{0}^{R_{i}} V\left(Z_{s}\right) \mathrm{d} s\right)\right] \cdot c(\omega) \leq c(\omega)^{i}
\end{aligned}
$$

where

$$
c(\omega):=\sup _{x \in \bar{B}(0)} E_{x}\left[\exp \left(-\int_{0}^{T_{D}} V\left(Z_{s}\right) \mathrm{d} s\right)\right] .
$$

We now argue that $\mathbb{P}$-a.s., $c(\omega)<1$. Note that a lower bound on the heat kernel in a region of $\mathbb{R}^{d}$ as the one obtained from ([37], Lem. 2.1) (or more generally [11], Thm. 3.3.5) is enough to deduce that given $\rho>0$ there is $\eta=\eta(\rho)>0$ such that for all measurable $A \subset B(0, \rho)$ and for all $x \in \bar{B}(0, \rho)$

$$
E_{x}\left[\int_{0}^{T_{B(0,2 \rho)}} \mathbf{1}_{A}\left(Z_{s}\right) \mathrm{d} s\right]>\eta \operatorname{Leb}(A) .
$$

Now let $A:=\{V(\cdot, \omega)>\varepsilon\} \cap B(u, \rho)$ and let $Y:=\int_{0}^{T_{B(0,2 \rho)}} \mathbf{1}_{A}\left(Z_{s}\right) \mathrm{d} s$. Then by (3.4), (1.8) and by CauchySchwarz, for all $x \in \bar{B}(u, \rho)$,

$$
\varepsilon \eta \leq E_{x}(Y) \leq E_{x}(Y ; Y>\varepsilon \eta / 2)+\varepsilon \eta / 2 \leq\left(E_{x}\left(Y^{2}\right) P_{x}(Y>\varepsilon \eta / 2)\right)^{1 / 2}+\varepsilon \eta / 2 .
$$


Hence for all $x \in \bar{B}(u, \rho)$, there is a constant $C>0$ such that

$$
P_{x}\left[\int_{0}^{T_{B(0,2 \rho)}} \mathbf{1}_{A}\left(Z_{s}\right) \mathrm{d} s>\varepsilon \eta / 2\right]>\left(\frac{\varepsilon \eta}{2}\right)^{2} \frac{1}{E_{x}\left(Y^{2}\right)}>\frac{C}{\rho^{4}}\left(\frac{\varepsilon \eta}{2}\right)^{2} .
$$

Moreover, by the tubular estimate ([37], p. 198), there is a positive constant $C$ such that for all $t>0$ and $x \in \bar{B}(0)$,

$$
\begin{aligned}
P_{x}\left[\sup _{0<s<t}\left|Z_{s}-\left(x+\frac{s}{t}(u-x)\right)\right|<\rho\right] & \geq C \exp \left(-\lambda_{d} \frac{t}{\rho^{2}}-\frac{1}{2 t}|u-x|^{2}\right) \\
& \geq C \exp \left(-\lambda_{d} \frac{t}{\rho^{2}}-\frac{1}{t}\left(|u|^{2}+1\right)\right) .
\end{aligned}
$$

Recall here that $\lambda_{d}$ is the principal Dirichlet eigenvalue of $-\frac{1}{2} \Delta$ in the unit ball. Hence, by taking $t=|u|+1$ in (3.6),

$$
\begin{aligned}
P_{x}\left[T_{D}>H_{B(u, \rho)}\right] & \geq P_{x}\left[\sup _{0<s<t}\left|Z_{s}-\left(x+\frac{s}{t}(u-x)\right)\right|<\rho\right] \\
& \geq C \exp \left(-\left(1+\frac{\lambda_{d}}{\rho^{2}}\right)(|u|+1)\right) .
\end{aligned}
$$

Then by (3.5), (3.7) and by the strong Markov property, for all $x \in \bar{B}(0), P_{x}\left[\int_{0}^{T_{D}} \mathbf{1}_{A}\left(Z_{s}\right) \mathrm{d} s>\eta \varepsilon / 2\right]$

$$
\begin{aligned}
& \geq P_{x}\left[T_{D}>H_{B(u, \rho)}, P_{Z_{H_{B(u, \rho)}}}\left(\int_{0}^{T_{B(u, 2 \rho)}} \mathbf{1}_{A}\left(Z_{s}\right) \mathrm{d} s>\eta \varepsilon / 2\right)\right] \\
& >C \exp \left(-\left(1+\frac{\lambda_{d}}{\rho^{2}}\right)(|u|+1)\right)\left(\frac{\varepsilon \eta}{2 \rho^{2}}\right)^{2}:=p_{0}(u) .
\end{aligned}
$$

This provides the following upper bound for $c(\omega)$ defined in (3.3).

$$
\begin{aligned}
c(\omega) & =\sup _{x \in \bar{B}(0)} E_{x}\left[\exp \left(-\int_{0}^{T_{D}} V\left(Z_{s}\right) \mathrm{d} s\right)\right] \\
& \leq \sup _{x \in \bar{B}(0)} E_{x}\left[\exp \left(-\varepsilon \int_{0}^{T_{D}} \mathbf{1}_{A}\left(Z_{s}\right) \mathrm{d} s\right)\right] \\
& \leq \exp \left(-\eta \varepsilon^{2} / 4\right) p_{0}(u)+1-p_{0}(u) \\
& =1-p_{0}(u)\left(1-e^{-\eta \varepsilon^{2} / 4}\right) .
\end{aligned}
$$

We now complete the proof of Lemma 2.4 when $d=1$ or 2. By (3.1) and (3.2), for all $x \in \bar{B}(0)$,

$$
\begin{aligned}
\int_{\bar{B}(0)} g(x, y, \omega) \mathrm{d} y & \leq C(|u|+2 \rho+1)^{2} \sum_{i=1}^{\infty} E_{x}\left[\exp \left(-\int_{0}^{R_{i}} V\left(Z_{s}\right) \mathrm{d} s\right)\right] \\
& \leq C(|u|+2 \rho+1)^{2} \frac{1}{1-c(\omega)} .
\end{aligned}
$$


Therefore by (3.8) and (3.9),

$$
\begin{aligned}
\log ^{+} \int_{B(0) \times B(0)} g(x, y, \omega) \mathrm{d} x \mathrm{~d} y & \leq C\left[1+\log ^{+}|u|-\log (1-c(\omega))\right] \\
& \leq C\left[1+\log ^{+}|u|-\log p_{0}(u)\right] \\
& \leq C\left[1+\log ^{+}|u|+|u|\right] .
\end{aligned}
$$

Since $\mathbb{E}\left(\log ^{+}|u|\right) \leq \mathbb{E}(|u|)<\infty$, (2.6) follows.

(2.7) follows from (3.10) and the fact that if $X(x), x \in \mathbb{Z}^{d}$ are identically distributed with $\mathbb{E}\left(|X(0)|^{d}\right)<\infty$, then $\lim _{|x| \rightarrow \infty} X(x) /|x|=0, \mathbb{P}$ - a.s. by Borel-Cantelli lemma.

Proof of Theorem 2.1. For a fixed $x \in \mathbb{R}^{d} \backslash\{0\}$, consider

$$
X_{s, r}:=d(s x, r x, \omega), \quad 0 \leq s \leq r
$$

where $d$ was defined in (2.5). We have that

(i) $X_{s, r} \leq X_{s, u}+X_{u, r}$ for all $0 \leq s \leq u \leq r$.

(ii) $X_{s, r} \circ \tau_{u x}=X_{s+u, r+u}$ for all $u \geq 0$.

Let $X_{[0,1]}:=\sup \left\{X_{s, r} ; 0 \leq s<r \leq 1\right\}$. The next step is to show that

(iii) $\mathbb{E}\left[X_{[0,1]}\right]<\infty$.

Let $z \in \mathbb{R}^{d}$ with $|z-x|>1$. Then for $\omega \in \Omega$ and $t>0$, we have that

$$
\begin{aligned}
e(z, x, \omega) & =E_{z}\left[\exp \left(-\int_{0}^{H(x)} V\left(Z_{s}, \omega\right) \mathrm{d} s\right), H(x)<\infty\right] \\
& \geq E_{z}\left[\exp \left(-\int_{0}^{t} V\left(Z_{s}, \omega\right) \mathrm{d} s\right), \sup _{0 \leq s \leq t} \mid Z_{s}-\left(z+\frac{s}{t}(x-z) \mid<1\right]\right. \\
& \geq P_{z}\left[\sup _{0 \leq s \leq t} \mid Z_{s}-\left(z+\frac{s}{t}(x-z) \mid\right)<1\right] \exp \left(-t \sup _{y \in \mathcal{C}_{1}(z, x)} V(y, \omega)\right)
\end{aligned}
$$

where, for $\rho>0, \mathcal{C}_{\rho}(z, x):=\left\{y \in \mathbb{R}^{d} ; \inf _{0 \leq s \leq 1}|y-(z+s(x-z))|<\rho\right\}$ is the interior of a cylinder of radius $\rho$.

By the tubular estimate ([37], p. 198), there exists a positive constant $C$ such that for all $t>0$ and $\rho>0$,

$$
P_{z}\left[\sup _{0 \leq s \leq t}\left|Z_{s}-\left(z+\frac{s}{t}(x-z)\right)\right|<\rho\right] \geq C \exp \left(-t \frac{\lambda_{d}}{\rho^{2}}-\frac{|x-z|^{2}}{2 t}\right) .
$$

Set $t=|x-z|$. Then by (3.11) and (3.12),

$$
-\log e(z, x, \omega) \leq-\log C+|x-z|\left(\lambda_{d}+\frac{1}{2}+\sup _{y \in \mathcal{C}_{1}(z, x)} V(y, \omega)\right) .
$$

Hence, there is a positive constant $C_{0}$ such that

$$
d(z, x) \leq C_{0}+(|x-z|+1)\left(\lambda_{d}+\frac{1}{2}+\sup _{y \in \mathcal{C}_{2}(z, x)} V(y, \omega)\right)
$$

and $\mathbb{E}\left(X_{[0,1]}\right) \leq C_{0}+(|x|+1) \mathbb{E}\left[\sup _{y \in \mathcal{C}_{2}(0, x)} V(y, \omega)\right]$ which is finite by stationarity of the potential and by $(2.2)$. 
By the continuous parameter subadditive theorem (see [20], Thm. 1.5.6) and since we assumed that the dynamical system is ergodic, there exists a constant $\alpha(x)$ such that $\mathbb{P}-$ a.s.

$$
\lim _{r \rightarrow \infty} \frac{1}{r} d(0, r x, \omega)=\lim _{r \rightarrow \infty} \frac{1}{r} \mathbb{E}[d(0, r x, \omega)]=\inf _{r>0} \frac{1}{r} \mathbb{E}[d(0, r x, \omega)]=\alpha(x) .
$$

It is easy to check that $\alpha(\cdot)$ is a semi-norm on $\mathbb{R}^{d}$.

By Lemmas 2.3 and $2.4, d(0, x, \omega)$ can be replaced by $a(0, x, \omega)$ or by $-\log g(0, x, \omega)$ in $(3.14)$.

Proof of Theorem 2.2. By stationarity of the potential and by translation invariance of Brownian motion, $d\left(x, y, \tau_{z} \omega\right)=d(x+z, y+z, \omega)$ for $x, y, z \in \mathbb{R}^{d}, \omega \in \Omega$. Moreover, by Lemma 5.2 .1 of $[37], d(\cdot, \cdot, \omega)$ is a.s. a distance on $\mathbb{R}^{d}$. Under the integrability condition (1.6), it follows from $(3.13)$ that $d(0, x, \omega)$ is in $L_{\mathbb{P}}(d, 1)$ for all $x \in \mathbb{Z}^{d}$.

Hence the conditions of the shape theorem ([2], Thm. 1.2) are satisfied. Therefore, there exists a semi-norm $L$ on $\mathbb{R}^{d}$ such that

$$
\lim _{|x| \rightarrow \infty, x \in \mathbb{Z}^{d}} \frac{d(0, x, \omega)-L(x)}{|x|}=0 \quad \text { a.s. }
$$

But by Theorem 2.1, $\alpha(x)=L(x)$ for all $x \in \mathbb{Z}^{d}$ and consequently, for all $x \in \mathbb{R}^{d}$.

For $x \in \mathbb{R}^{d}$, denote by $\hat{x}$ the nearest neighbor point in $\mathbb{Z}^{d}$ of $x$ (with some rule to break ties). Then, $|x-\hat{x}|<$ $\sqrt{d}$ and for all $x \in \mathbb{R}^{d} \backslash B(0)$,

$$
\begin{aligned}
\frac{|d(0, x, \omega)-\alpha(x)|}{|x|} & \leq \frac{|d(0, x, \omega)-d(0, \hat{x}, \omega)|}{|x|}+\frac{|d(0, \hat{x}, \omega)-\alpha(\hat{x})|}{|x|}+\frac{|\alpha(\hat{x})-\alpha(x)|}{|x|} \\
& \leq \frac{|d(\hat{x}, x, \omega)|}{|\hat{x}|} \cdot \frac{|\hat{x}|}{|x|}+\frac{|d(0, \hat{x}, \omega)-\alpha(\hat{x})|}{|\hat{x}|} \cdot \frac{|\hat{x}|}{|x|}+\frac{\alpha(x-\hat{x})}{|x|}
\end{aligned}
$$

Consider successively the terms on the right hand side of (3.16) above. As in (3.13), for all $x \in \mathbb{R}^{d}$,

$$
\begin{aligned}
d(\hat{x}, x, \omega) & \leq C_{0}(|\hat{x}-x|+1)+\sup _{y \in \mathcal{C}_{2}(\hat{x}, x)} V(y, \omega) \\
& \leq C_{0}(\sqrt{d}+1)+\sup _{B(\hat{x}, \sqrt{d}+3)} V(\cdot, \omega) .
\end{aligned}
$$

Let $Y(\hat{x}):=C_{0}(\sqrt{d}+1)+\sup _{B(\hat{x}, \sqrt{d}+3)} V(\cdot, \omega)$. Since $\left(Y(\hat{x}), \hat{x} \in \mathbb{Z}^{d}\right)$ are identically distributed and in $L_{\mathbb{P}}^{d}$, by Borel-Cantelli lemma,

$$
\lim _{x \rightarrow \infty} \frac{d(\hat{x}, x)}{|\hat{x}|} \cdot \frac{|\hat{x}|}{|x|} \leq \lim _{\hat{x} \rightarrow \infty, \hat{x} \in \mathbb{Z}^{d}} \frac{2 Y(\hat{x})}{|\hat{x}|}=0 \quad \mathbb{P} \text { - a.s. }
$$

So the first term of (3.16) converges a.s. to 0. From (3.15) and from (2.8) respectively, the second and third terms converge to 0 a.s. Hence, $\mathbb{P}$ - a.s.,

$$
\lim _{|x| \rightarrow \infty, x \in \mathbb{R}^{d}} \frac{1}{|x|}|d(0, x, \omega)-\alpha(x)|=0
$$

By using (2.7) and Lemma 2.3, $d(0, x)$ can be replaced by $a(0, x)$ or $-\log g(0, x)$ in $(3.17)$.

Proof of Lemma 2.5. The lower bound of (2.8) is proved as in ([37], Prop. 2.9). Let $\widetilde{V}:=V-\underline{V}$. Then for $x \in \mathbb{R}^{d},|x|>1$ 


$$
\begin{aligned}
e_{\lambda}(0, x, \omega) & =E_{0}\left[\exp \left[-\int_{0}^{H(x)}\left(\lambda+V\left(Z_{s}, \omega\right) \mathrm{d} s\right)\right], H(x)<\infty\right] \\
& =E_{0}\left[\exp (-(\lambda+\underline{V}) H(x)) \exp \left(-\int_{0}^{H(x)} \widetilde{V}\left(Z_{s}, \omega\right) \mathrm{d} s\right), H(x)<\infty\right] \\
& \leq \exp (-\sqrt{2(\lambda+\underline{V})}|x|)
\end{aligned}
$$

since for a one-dimensional Brownian motion, for $\lambda \geq 0$ and $y \in \mathbb{R}, E_{0}[\exp (-\lambda H(y))]=\exp (-\sqrt{2 \lambda}|y|)$.

To prove the upper bound of (2.8), note that for $\lambda \geq-\underline{V},|y|>1$ and for any $t>0$,

$$
\begin{aligned}
e_{\lambda}(0, y, \omega) & =E_{0}\left[\exp \left(-\int_{0}^{H(y)}\left(\lambda+V\left(Z_{s}, \omega\right)\right) \mathrm{d} s\right), H(y)<\infty\right] \\
& =E_{0}\left[\exp \left(-\int_{0}^{H(y)}\left(\lambda+\underline{V}+\widetilde{V}\left(Z_{s}, \omega\right)\right) \mathrm{d} s\right), H(y)<\infty\right] \\
& \geq P_{0}\left[\sup _{0 \leq s \leq t}\left|Z_{s}-\frac{s}{t} y\right|<1\right] \exp \left(-\lambda t-\underline{V} t-\int_{0}^{t} h\left(\frac{s}{t} y, \omega\right) \mathrm{d} s\right)
\end{aligned}
$$

where $h(z, \omega):=\sup _{B(z)} \tilde{V}(\cdot, \omega), z \in \mathbb{R}^{d}$.

Then by the tubular estimate [37, p.198] and by the stationarity of $V$, for $\lambda \geq-\underline{V},|y|>1$ and $t>0$,

$$
\begin{aligned}
-\mathbb{E} \log e_{\lambda}(0, y, \omega) & \leq-\log P_{0}\left[\sup _{0 \leq s \leq t}\left|Z_{s}-\frac{s}{t} y\right|<1\right]+(\lambda+\underline{V}) t+\int_{0}^{t} \mathbb{E} h\left(\frac{s}{t} y, \omega\right) \mathrm{d} s \\
& \leq C_{0}+\lambda_{\mathrm{d}} t+\frac{|y|^{2}}{2 t}+(\lambda+\underline{V}) t+t \mathbb{E} h(0, \cdot) \\
& =C_{0}+\left(\lambda_{d}+\lambda+\mathbb{E}\left[\sup _{B(0)} V\right]\right) t+\frac{|y|^{2}}{2 t} .
\end{aligned}
$$

Let $y=n x$ and $t=\frac{n|x|}{\sqrt{2\left(\lambda+\lambda_{d}+\mathbb{E}\left[\sup _{B(0)} V\right]\right.}}$ to obtain (2.8).

Since $\lambda \mapsto \alpha_{\lambda}(x)$ is a concave function on $[-\underline{V}, \infty[$ for $\lambda>-\underline{V}$,

$$
\alpha_{\lambda}^{\prime}(x)_{-} \leq \frac{\alpha_{\lambda}(x)-\alpha_{-\underline{V}}(x)}{\lambda} \leq \frac{\alpha_{\lambda}(x)}{\lambda} .
$$

And by (2.8), $\alpha_{\lambda}(x) / \lambda \rightarrow 0$ as $\lambda \rightarrow \infty$. (2.9) follows.

To prove the continuity and concavity properties of $\alpha_{\lambda}$ one can then proceed as in ([37], Sect. 5.2).

\subsection{Proof of the upper estimate (1.9)}

We follow the arguments of ([37], (4.6) of Thm. 5.4.2). See also ([45], (69) of Thm. 19).

First assume that $A$ is a compact subset of $\mathbb{R}^{d}$. For each $t>0$, it is possible to choose $n_{t}$ points $x_{1}, x_{2}, \ldots, x_{n_{t}}$ in $A$ such that $n_{t}$ grows at most polynomially in $t$ and $t A \subset B_{t}:=\cup_{k=1}^{n_{t}} B\left(x_{k}\right)$. By definitions of $S_{t, \omega}$ and $Q_{t, \omega}$, $\mathbb{P}$ - a.s. for all $\lambda \geq 0, \exp (-\lambda t) S_{t, \omega} Q_{t, \omega}\left(Z_{t} \in t A\right)$

$$
\begin{aligned}
& =\exp (-\lambda t-\underline{V} t) E_{0}\left[\exp \left(-\int_{0}^{t}(V-\underline{V})\left(Z_{s}, \omega\right) \mathrm{d} s\right), Z_{t} \in t A\right] \\
& \leq \exp (-\lambda t-\underline{V} t) \sum_{k=1}^{n_{t}} E_{0}\left[\exp \left(-\int_{0}^{t}(V-\underline{V})\left(Z_{s}, \omega\right) \mathrm{d} s\right), Z_{t} \in B\left(x_{k}\right)\right]
\end{aligned}
$$




$$
\begin{aligned}
= & \exp (-\underline{V} t) \sum_{k=1}^{n_{t}} E_{0}\left[\exp \left(-\int_{0}^{t}(\lambda+V-\underline{V})\left(Z_{s}, \omega\right) \mathrm{d} s\right), Z_{t} \in B\left(x_{k}\right)\right] \\
\leq & \exp (-\underline{V} t) \sum_{k=1}^{n_{t}} E_{0}\left[\exp \left(-\int_{0}^{H\left(x_{k}\right)}(\lambda+V-\underline{V})\left(Z_{s}, \omega\right) \mathrm{d} s\right), H\left(x_{k}\right)<\infty\right] \\
& \quad \text { ince } \lambda+V-\underline{V} \geq 0 \\
= & \exp (-\underline{V} t) \sum_{k=1}^{n_{t}} e_{\lambda-\underline{V}}\left(0, x_{k}, \omega\right) \leq \exp (-\underline{V} t) n_{t} \max _{1 \leq k \leq n_{t}} e_{\lambda-\underline{V}}\left(0, x_{k}, \omega\right) .
\end{aligned}
$$

Therefore for all $\lambda \geq 0$, by Theorem 2.2, (1.5) and under the assumption that $\lambda_{V}=\underline{V}$,

$$
\begin{gathered}
-\lambda-\lambda_{V}+\limsup _{t \rightarrow \infty} \frac{1}{t} \log Q_{t, \omega}\left(Z_{t} \in t A\right) \leq-\underline{V}-\inf _{A} \alpha_{\lambda-\underline{V}}(x), \quad \mathbb{P}-\text { a.s. } \\
\text { Hence } \limsup _{t \rightarrow \infty} \frac{1}{t} \log Q_{t, \omega}\left(Z_{t} \in t A\right) \leq-\sup _{\lambda \geq 0} \inf _{x \in A}\left(\alpha_{\lambda-\underline{V}}(x)-\lambda\right) .
\end{gathered}
$$

To complete the proof, it remains to interchange the sup and the inf in (3.18). This is done by a classical argument (see for example [13] or [37], p. 250). It does not require additional properties of the potential. Neither does the proof of the general case when $A$ is a closed subset of $\mathbb{R}^{d}$ as can be seen from ([37], p. 250).

\subsection{Proof of the lower estimate (1.10)}

The following lemmas will be needed.

Denote by $[x, y]$, the line segment between the vertices $x, y$ of $\mathbb{R}^{d}$. For $y \in \mathbb{R}^{d} \backslash\{0\}$, let $\Pi(y)$ be the hyperplane orthogonal to $y$ which contains $y / 2$ and let $\mathcal{S}(y):=\left\{\xi \in \mathbb{R}^{d} ; \xi=z /|z|\right.$ for some $z \in \Pi(y)$ with $\left.|z|<|y|\right\}$. For $\xi \in \mathcal{S}(y)$, denote by $[y, \xi, 0]$, the broken line from $y$ to 0 consisting of the line segments $[y, z]$ and $[z, 0]$ where $z \in \Pi(y)$ is such that $\xi=z /|z|$. The path integral of a measurable function $f: \mathbb{R}^{d} \rightarrow \mathbb{R}$ along the broken line $[y, \xi, 0]$ is denoted by

$$
\int_{[y, \xi, 0]} f:=\int_{0}^{1} f(y+r(z-y))|z| d r+\int_{0}^{1} f(r z)|z| d r .
$$

By ([6], Prop. 3), if $h_{0}(\cdot) \in L_{\mathbb{P}}(d, 1)$ then, $\mathbb{P}$ - a.s., $h(x, \omega):=h_{0}\left(\tau_{x} \omega\right)$ is locally in $L_{\mathbb{R}^{d}}(d, 1)$, the Lorentz space over $\mathbb{R}^{d}$ with respect to the Lebesgue measure, (see also [3], pp. 31-32). This in turn implies that $\mathbb{P}$ - a.s., the function $\mathcal{M} h(y, \omega)$ defined by

$$
\mathcal{M} h(y, \omega):=\frac{1}{\sigma(\mathcal{S}(y))} \int_{\mathcal{S}(y)} d \sigma(\xi) \int_{[0, \xi, y]} h,
$$

where $\sigma(\cdot)$ denotes the Lebesgue (area) measure on the unit sphere of $\mathbb{R}^{d}$, is continuous on $\mathbb{R}^{d}$. Note that $\sigma(\mathcal{S}(y))$ does not depend on $y \in \mathbb{R}^{d} \backslash\{0\}$.

Then the arguments given in the proof of ([3], Thm. 7) apply to $\mathcal{M} h(y, \omega)$. They lead to the following maximal inequality.

Lemma 3.1. Let $h_{0} \in L_{\mathbb{P}}(d, 1)$. Then there is a positive constant $c_{3}$ such that for $m>0$,

$$
\mathbb{P}\left(\sup _{|y|>1} \frac{1}{|y|} \mathcal{M} h(y, \omega)>m\right)<c_{3} m^{-d}\left\|h_{0}\right\|_{d, 1}^{d}
$$

where $h(x, \omega):=h_{0}\left(\tau_{x} \omega\right)$. 
Lemma 3.2. Let $U: \mathbb{R}^{d} \rightarrow\left[0, \infty\left[\right.\right.$ be a measurable function. Then there are positive constants $c_{4}$ and $c_{5}$ such that for $y \in \mathbb{R}^{d},|y|>1, t>0$ and $\xi \in \mathcal{S}(y)$,

$$
\begin{aligned}
& E_{y}\left[\exp \left(-\int_{0}^{H(0)} U\left(Z_{s}\right) \mathrm{d} s\right), H(0) \leq t\right] \\
& \quad \geq c_{4} \exp \left[-\lambda_{d} t-c_{5} \frac{|y|^{2}}{t}-\frac{t}{|y|} \int_{[y, \xi, 0]} M_{2}\right]
\end{aligned}
$$

where $M_{2}(z):=\sup _{x \in B(z, 2)} U(x)$.

Proof. It is possible to generalize the argument used in the proof of (2.8) to any broken line $[y, \xi, 0]$ by combining the tubular estimates ([37], p. 198) with the strong Markov property as follows.

Let $z \in \Pi(y)$ be such that $z /|z|=\xi$ and $|z|<|y|$. Let $M_{R}(z):=\sup _{x \in B(z, R)} U(x), R>0$. Then

$$
\begin{aligned}
E_{y} & {\left[\exp \left(-\int_{0}^{H(0)} U\left(Z_{s}\right) \mathrm{d} s\right), H(0) \leq 2 t\right] } \\
\geq & P_{y}\left[\sup _{0 \leq s \leq t}\left|Z_{s}-y+\frac{s}{t}(y-z)\right|<1\right] \exp \left[-\int_{0}^{t} M_{1}\left(y-\frac{s}{t}(y-z)\right) \mathrm{d} s\right] \\
& \times \inf _{z^{\prime} \in B(z)} P_{z^{\prime}}\left[\sup _{0 \leq s \leq t}\left|Z_{s}-z^{\prime}+\frac{s}{t} z^{\prime}\right|<1\right] \exp \left[-\int_{0}^{t} M_{2}\left(z-\frac{s}{t} z\right) \mathrm{d} s\right] \\
\geq & C \exp \left[-\lambda_{d} t-\frac{1}{2 t}|y-z|^{2}-\int_{0}^{t} M_{2}\left(y-\frac{s}{t}(y-z)\right) \mathrm{d} s\right] \\
& \times \inf _{z^{\prime} \in B(z)} C \exp \left[-\lambda_{d} t-\frac{1}{2 t}\left|z^{\prime}\right|^{2}-\int_{0}^{t} M_{2}\left(z-\frac{s}{t} z\right) \mathrm{d} s\right] \\
\geq & C \exp \left[-2 \lambda_{d} t-\frac{1}{2 t}\left(|y-z|^{2}+\left|z^{\prime}\right|^{2}\right)-\frac{t}{|y-z|} \int_{[y, z]} M_{2}-\frac{t}{|z|} \int_{[z, 0]} M_{2}\right] \\
\geq & C \exp \left[-2 \lambda_{d} t-\frac{C^{\prime}}{t}|y|^{2}-\frac{t}{|z|} \int_{[y, \xi, 0]} M_{2}\right]
\end{aligned}
$$

since $M_{R} \geq 0$ and by using the inequalities

$$
\frac{1}{2} \leq \frac{|y|}{2} \leq|z|=|z-y| \leq|y| \text { and }\left|z^{\prime}-z\right| \leq 1
$$

Lemma 3.3. Let $A$ be an event such that $\mathbb{P}(A)>1-\varepsilon$ for some $\varepsilon \in] 0,1 / 2\left[\right.$. Let $v \in \mathbb{R}^{d} \backslash\{0\}$.

Then $\mathbb{P}-$ a.s. for all $\delta>\frac{2 \varepsilon}{1-2 \varepsilon}$ and for all sufficiently large $t$, there is $\left.s \in\right] t,(1+\delta) t\left[\right.$ such that $\tau_{\text {sv }} \omega \in A$.

Proof. By the ergodic theorem, a.s. and for all $t$ sufficiently large,

$$
\begin{aligned}
1-2 \varepsilon<\mathbb{P}(A)-\varepsilon & \leq \frac{1}{(1+\delta) t} \int_{0}^{(1+\delta) t} \mathbf{1}_{A}\left(\tau_{s v} \omega\right) \mathrm{d} s \\
& \leq \frac{1}{1+\delta}+\frac{1}{(1+\delta) t} \int_{t}^{(1+\delta) t} \mathbf{1}_{A}\left(\tau_{s v} \omega\right) \mathrm{d} s
\end{aligned}
$$

Hence if $(1-2 \varepsilon)(1+\delta)-1>0$ then $\int_{t}^{(1+\delta) t} \mathbf{1}_{A}\left(\tau_{s v} \omega\right) \mathrm{d} s>0$. 
The principal Dirichlet eigenvalue of $-\frac{1}{2} \Delta+V$ in the ball $B(0, R), R>0$, is defined as

$$
\lambda_{V, \omega}(B(0, R)):=\inf \left\{\int_{\mathbb{R}^{d}}\left(\frac{1}{2}|\nabla \varphi|^{2}+V \varphi^{2}\right) \mathrm{d} x ; \varphi \in \mathcal{C}_{c}^{\infty}(B(0, R)), \int_{\mathbb{R}^{d}} \varphi^{2} \mathrm{~d} x=1\right\} .
$$

From definitions (1.4) and (3.19), it is clear that

$$
\lambda_{V, \omega}(B(0, R)) \downarrow \lambda_{V}\left(\mathbb{R}^{d}\right) \text { a.s. as } R \rightarrow \infty .
$$

Similarly to $(1.5), \lambda_{V, \omega}(B(0, R))$ is related to the survival time in $B(0, R)$. We will need the following version of ([37], (3.1.17)) where an $\inf _{z \in B(0)}$ appears. The argument does not require a Harnack-type inequality.

Lemma 3.4. Let $V$ be a non-negative, stationary and ergodic potential which satisfies (1.6) and (1.8). Then $\mathbb{P}$ - a.s. for all $R>2$,

$$
\liminf _{t} \frac{1}{t} \log \inf _{z \in B(0)} E_{z}\left[\exp \left(-\int_{0}^{t} V\left(Z_{s}, \omega\right) \mathrm{d} s\right), T_{B(0, R)}>t\right] \geq-\lambda_{V, \omega}(B(0, R)) .
$$

Proof. Note that a stationary ergodic potential $V$ which satisfies (2.2) and (1.8), also belongs to $\mathcal{K}_{d}^{\text {loc }}$ and proceed as in ([37], Sect. 3.1).

Fix $R>2$. For $\eta>0$, let $\varphi \in \mathcal{C}_{c}^{\infty}(B(0, R)), \varphi \geq 0$, be such that

$$
\lambda_{V, \omega}(B(0, R)) \leq \int_{\mathbb{R}^{d}}\left(\frac{1}{2}|\nabla \varphi|^{2}+V \varphi^{2}\right) \mathrm{d} x \leq \lambda_{V, \omega}(B(0, R))+\eta .
$$

Let $r_{R}(t, x, y, \omega)$ be the transition density of the Brownian motion in the potential $V(\cdot, \omega)-\underline{V}$ killed when exiting $B(0, R)$. Then for $z \in B(0, R)$,

$$
\begin{aligned}
& \exp \left(-t\left(\lambda_{V, \omega}(B(0, R))+\eta\right)\right) \\
& \leq \iint \varphi(x) e^{-t \underline{V}} r_{R}(t, x, y) \varphi(y) \mathrm{d} x \mathrm{~d} y, \quad \text { by Jensen's inequality, } \\
& \leq e^{\underline{V}} \iint \varphi(x) e^{-(t+1) \underline{V}} \frac{r_{R}(1, z, x)}{\inf _{\operatorname{supp} \varphi} r_{R}(1, z, \cdot)} r_{R}(t, x, y) \varphi(y) \mathrm{d} x \mathrm{~d} y \\
& \leq \frac{\|\varphi\|_{\infty}^{2}}{\inf _{\operatorname{supp} \varphi} r_{R}(1, z, \cdot)} e^{\underline{V}} E_{z}\left[\exp \left(-\int_{0}^{t+1} V\left(Z_{s}, \omega\right) \mathrm{d} s\right), T_{B(0, R)}>t+1\right] \\
& \leq \frac{\|\varphi\|_{\infty}^{2}}{\inf _{z \in B(0)} \inf _{\operatorname{supp} \varphi} r_{R}(1, z, \cdot)} e^{\underline{V}} \sup _{z \in B(0)} E_{z}\left[\exp \left(-\int_{0}^{t+1} V\left(Z_{s}, \omega\right) \mathrm{d} s\right), T_{B(0, R)}>t+1\right] .
\end{aligned}
$$

By ([37], Prop. 1.3.5) and ([37], Lem. 2.2.1) $r_{R}(1, \cdot, \cdot)$ is continuous and strictly positive in $B(0, R)$. Since $\operatorname{supp} \varphi$ is compact, $\inf _{z \in B(0)} \inf _{\operatorname{supp} \varphi} r_{R}(1, z, \cdot)>0$. The result follows.

A close examination of the proof of the following key lemma from [36] or [37], shows that it holds for stationary potentials under the moment condition (2.2).

For $v \in \mathbb{R}^{d} \backslash\{0\}, 0<s_{1}<s_{2}<\infty, 0 \leq m \leq n$, define

$$
\begin{aligned}
S_{m, n, v, s_{1}} & :=H(n v) \circ \theta_{(n-m) s_{1}}+(n-m) s_{1}, \\
A_{m, n, v, s_{1}, s_{2}} & :=\left\{S_{m, n, v, s_{1}}<(n-m) s_{2}\right\}
\end{aligned}
$$


where $\theta_{t}, t \geq 0$ is the canonical shift on $\mathcal{C}\left(\mathbb{R}^{+}, \mathbb{R}^{d}\right)$. Note that $S_{m, n, v, s_{1}}$ is a stopping time and $A_{m, n, v, s_{1}, s_{2}}$ is the event that $Z$. enters $B(n v)$ in the time interval $\left[(n-m) s_{1},(n-m) s_{2}\right]$. Define $b_{\lambda}\left(m, n, v, s_{1}, s_{2}, \omega\right)$ by

$$
-\inf _{z \in B(m v)} \log E_{z}\left[\exp \left(-\int_{0}^{S_{m, n, v, s_{1}}}(\lambda+V)\left(Z_{s}, \omega\right) \mathrm{d} s\right), A_{m, n, v, s_{1}, s_{2}}\right] .
$$

The strong Markov property implies that $\left\{b_{\lambda}\left(m, n, v, s_{1}, s_{2}, \omega\right)\right\}_{m \geq 0, n \geq 0}$ is a subadditive sequence. A calculation similar to (3.13) shows that $\mathbb{E} b_{\lambda}\left(0,1, v, s_{1}, s_{2}\right)<\infty$.

Lemma 3.5. ([37], Lem. 5.4.3) Let $V$ be a non-negative, stationary and ergodic potential which satisfies (1.6) and (1.8). Then for $v \in \mathbb{R}^{d}, v \neq 0, \lambda>0,0<s_{1}<s_{2}<\infty$, P-a.s.

$$
\lim _{n \rightarrow \infty} \frac{b_{\lambda}\left(0, n, v, s_{1}, s_{2}, \omega\right)}{n}=\lim _{n \rightarrow \infty} \mathbb{E} \frac{b_{\lambda}\left(0, n, v, s_{1}, s_{2}, \omega\right)}{n}:=\kappa_{\lambda}\left(v, s_{1}, s_{2}\right) \in[0, \infty)
$$

Moreover, if $\lambda>0$ and $] s_{1}, s_{2}\left[\cap\left[\alpha_{\lambda}^{\prime}(v)_{+}, \alpha_{\lambda}^{\prime}(v)_{-}\right] \neq \emptyset\right.$ then

$$
\kappa_{\lambda}\left(v, s_{1}, s_{2}\right) \leq \alpha_{\lambda}(v) .
$$

Here $\alpha_{\lambda}^{\prime}(v)_{+}, \alpha_{\lambda}^{\prime}(v)_{-}$are respectively the right and left derivatives of $\alpha_{\lambda}(v)$. To prove (3.22), use (2.9) and proceed as in ([37], Lem. 5.4.3). (3.21) follows from Kingman's subadditive ergodic theorem.

Note that for all $0 \leq s_{1}<s_{2}<\infty$ and $\lambda>0, \kappa_{\lambda}\left(v, s_{1}, s_{2}\right) \geq \alpha_{\lambda}(v)$.

Proof of (1.10). Let $\widetilde{V}:=V-\underline{V}$ and denote the corresponding Lyapunov exponents by $\widetilde{\alpha}_{\lambda}(x)$. Then $\widetilde{\alpha}_{\lambda}(x)=$ $\alpha_{\lambda-\underline{V}}(x)$ and under the assumption that $\lambda_{V}=\underline{V}$, we have that

$$
\lambda_{\widetilde{V}}=0 .
$$

Since for any open set $\mathcal{O} \subset \mathbb{R}^{d}$,

$$
S_{t, \omega} Q_{t, \omega}\left(Z_{t} \in t \mathcal{O}\right)=\exp (-\underline{V} t) E_{0}\left[\exp \left(-\int_{0}^{t}(V-\underline{V})\left(Z_{s}, \omega\right) \mathrm{d} s\right), Z_{t} \in t \mathcal{O}\right]
$$

and by the continuity of $I(\cdot)$, to obtain (1.10), it is sufficient to show that for all $v \in \mathbb{Q}^{d} \backslash\{0\}$ and $r>0$,

$$
\liminf _{t \rightarrow \infty} \frac{1}{t} \log E_{0}\left[\exp \left(-\int_{0}^{t} \widetilde{V}\left(Z_{s}, \omega\right) \mathrm{d} s\right), Z_{t} \in t B(v, r)\right] \geq-I(v) \quad \mathbb{P} \text { - a.s. }
$$

where $I(v)=\sup _{\lambda>0}\left(\widetilde{\alpha}_{\lambda}(v)-\lambda\right)$ as in $(2.10)$.

Fix $v \in \mathbb{Q}^{d} \backslash\{0\}$ and $r>0$ and consider the following events. For positive numbers $t_{0}, R, \varepsilon$, let $\mathcal{A}_{1}\left(t_{0}, R, \varepsilon\right)$ be the event

$$
\left\{\text { for all } t>t_{0}, \frac{1}{t} \log \inf _{z \in B(0)} E_{z}\left[\exp \left(-\int_{0}^{t} \widetilde{V}\left(Z_{s}, \omega\right) \mathrm{d} s\right), t<T_{B(0, R)}\right]>-\lambda_{\widetilde{V}}-\varepsilon\right\}
$$

and for $m>0$, let

$$
\begin{array}{r}
\mathcal{A}_{2}(m):=\{\text { for all } y,|y|>1, \text { there is a broken line }[0, \xi, y] \text { from } 0 \text { to } y \\
\text { with } \left.\xi \in \mathcal{S}(y) \text { and such that } \int_{[0, \xi, y]} M_{2}<m|y|\right\}
\end{array}
$$

where $M_{2}(x, \omega):=\sup _{B(x, 2)} \tilde{V}(\cdot, \omega)$ for $x \in \mathbb{R}^{d}$.

Finally, let $\Omega^{\prime}$ be the event of probability 1 where (3.21) holds. 
For the moment, assume that for some positive numbers $\left.\varepsilon^{\prime} \in\right] 0,1 / 4\left[, t_{0}, R, \varepsilon\right.$ and $m$,

$$
\mathbb{P}\left(\mathcal{A}_{1}\left(t_{0}, R, \varepsilon\right) \cap \mathcal{A}_{2}(m) \cap \Omega^{\prime}\right)>1-\varepsilon^{\prime} .
$$

Let $\omega \in \mathcal{A}_{1}\left(t_{0}, R, \varepsilon\right) \cap \mathcal{A}_{2}(m) \cap \Omega^{\prime}$. If $4 \varepsilon^{\prime}<\delta<r$ then $\delta>2 \varepsilon^{\prime} /\left(1-2 \varepsilon^{\prime}\right)$ and by Lemma 3.3 , for all $t$ sufficiently large there is $y_{t} \in \mathbb{R}^{d}$ such that

$$
3<\left|[t] v-y_{t}\right|<\delta t|v| \text { and } \tau_{y_{t}} \omega \in \mathcal{A}_{1}\left(t_{0}, R, \varepsilon\right) \cap \mathcal{A}_{2}(m) .
$$

Moreover, let $0<s_{1}<s_{2}<1$ and $\lambda>0$ be such that $] s_{1}, s_{2}\left[\cap\left[\widetilde{\alpha}_{\lambda}^{\prime}(v)_{+}, \widetilde{\alpha}_{\lambda}^{\prime}(v)_{-}\right] \neq \emptyset\right.$.

Then for $\eta \in] 0,1-s_{1}[$ and for all $t$ sufficiently large so that $R+\delta t<r t$,

$$
\begin{aligned}
E_{0}\left[\exp \left(-\int_{0}^{t} \widetilde{V}\left(Z_{s}, \omega\right) \mathrm{d} s\right), Z_{t} \in t B(v, r)\right] \\
\geq E_{0}\left[\exp \left(-\int_{0}^{S_{0,[t], v, s_{1}}} \widetilde{V}\left(Z_{s}, \omega\right) \mathrm{d} s\right), A_{0,[t], v, s_{1}, s_{2}}\right] \\
\quad \times \inf _{y \in B([t] v)} E_{y}\left[\exp \left(-\int_{0}^{H\left(y_{t}\right)} \widetilde{V}\left(Z_{s}, \omega\right) \mathrm{d} s\right), H\left(y_{t}\right) \leq \eta t\right] \\
\quad \times \inf _{z \in B\left(y_{t}\right)} E_{z}\left[\exp \left(-\int_{0}^{\left(1-s_{1}\right) t} \widetilde{V}\left(Z_{s}, \omega\right) \mathrm{d} s\right), T_{B\left(y_{t}, R\right)}>\left(1-s_{1}\right) t\right] .
\end{aligned}
$$

By Lemma 3.5 , since $\omega \in \Omega^{\prime}$ and $S_{0,[t], v, s_{1}} \geq[t] s_{1}$,

$$
\begin{aligned}
& \liminf \frac{1}{t} \log E_{0}\left[\exp \left(-\int_{0}^{S_{0,[t], v, s_{1}}} \widetilde{V}\left(Z_{s}, \omega\right) \mathrm{d} s\right), A_{0,[t], v, s_{1}, s_{2}}\right] \\
& \geq-\widetilde{\kappa}_{\lambda}(v)+\lambda s_{1} \geq-\widetilde{\alpha}_{\lambda}(v)+\lambda s_{1} .
\end{aligned}
$$

By Lemma 3.2 and since $\tau_{y_{t}} \omega \in \mathcal{A}_{2}(m)$, for some $\xi \in \mathcal{S}\left([t] v-y_{t}\right)$,

$$
\begin{aligned}
\inf _{y \in B([t] v)} E_{y} & {\left[\exp \left(-\int_{0}^{H\left(y_{t}\right)} \tilde{V}\left(Z_{s}, \omega\right) \mathrm{d} s\right), H\left(y_{t}\right) \leq \eta t\right] } \\
& \geq c_{4} \exp \left[-\lambda_{d} \eta t-\frac{c_{5}}{\eta t}\left|[t] v-y_{t}\right|^{2}-\frac{\eta t}{\left|[t] v-y_{t}\right|} \int_{y_{t}+\left[[t] v-y_{t}, \xi, 0\right]} M_{2}\right] \\
& \geq c_{4} \exp \left[-\lambda_{d} \eta t-\frac{c_{5}}{\eta t}\left|[t] v-y_{t}\right|^{2}-\eta t m\right]
\end{aligned}
$$

Hence

$$
\begin{aligned}
\liminf & \frac{1}{t} \log \inf _{y \in B([t] v)} E_{y}\left[\exp \left(-\int_{0}^{H\left(y_{t}\right)} \widetilde{V}\left(Z_{s}, \omega\right) \mathrm{d} s\right), H\left(y_{t}\right) \leq \eta t\right] \\
& \geq 0-\lambda_{d} \eta-c_{6} \frac{\delta^{2}}{\eta}|v|^{2}-\eta m .
\end{aligned}
$$

For the third term, since $\tau_{y_{t}} \omega \in \mathcal{A}_{1}\left(t_{0}, R, \varepsilon\right)$, by (3.23), whenever $\left(1-s_{1}\right) t>t_{0}$,

$$
\begin{aligned}
& \log \inf _{z \in B\left(y_{t}\right)} E_{z}\left[\exp \left(-\int_{0}^{T_{B\left(y_{t}, R\right)}} \widetilde{V}\left(Z_{s}, \omega\right) \mathrm{d} s\right), T_{B\left(y_{t}, R\right)}>\left(1-s_{1}\right) t\right] \\
& \quad \geq-\left(1-s_{1}\right)\left(\lambda_{\widetilde{V}}+\varepsilon\right) t=-\left(1-s_{1}\right) t \varepsilon .
\end{aligned}
$$


Putting together equations (3.26)-(3.29), we find that on $\mathcal{A}_{1}\left(t_{0}, R, \varepsilon\right) \cap \mathcal{A}_{2}(m) \cap \Omega^{\prime}$,

$$
\begin{gathered}
\underset{t}{\liminf } \frac{1}{t} \log E_{0}\left[\exp \left(-\int_{0}^{t} \widetilde{V}\left(Z_{s}, \omega\right) \mathrm{d} s\right), Z_{t} \in t B(v, r)\right] \\
\geq-\widetilde{\alpha}_{\lambda}(v)+\lambda s_{1}-\lambda_{d} \eta-c_{6} \frac{\delta^{2}}{\eta}|v|^{2}-\eta m-\left(1-s_{1}\right) \varepsilon .
\end{gathered}
$$

The proof will now be completed by contradiction. Assume that for some $v \in \mathbb{Q}^{d} \backslash\{0\}$ and for some positive numbers $r, \varepsilon_{0}$ and $\varepsilon_{1}$, on an event of probability greater than $\varepsilon_{0}$

$$
\liminf \frac{1}{t} \log E_{0}\left[\exp \left(-\int_{0}^{t} \widetilde{V}\left(Z_{s}, \omega\right) \mathrm{d} s\right), Z_{t} \in t B(v, r)\right]<-I(v)-\varepsilon_{1} .
$$

Set

$$
\varepsilon^{\prime}=\varepsilon^{\prime}(m):=2 c_{3} m^{-d}\left\|h_{0}\right\|_{d, 1}^{d}, \eta=\eta(m):=\frac{\varepsilon_{1}}{20 m} \text { and } \delta=\delta(m):=5 \varepsilon^{\prime} .
$$

Then for all $m$ positive, $\eta m<\varepsilon_{1} / 10$ and $4 \varepsilon^{\prime}<\delta$. Now, choose $m$ sufficiently large so that

$$
\varepsilon^{\prime}<\min \left\{1 / 4, \varepsilon_{0}\right\}, \eta<\min \left\{\varepsilon_{1} / 10 \lambda_{d}, 1-s_{1}\right\}, \delta<r \text { and } c_{6} \frac{\delta^{2}}{\eta} v^{2}<\varepsilon_{1} / 10 .
$$

Furthermore, choose $\varepsilon<\varepsilon_{1} / 10$. By (3.20) and by Lemma 3.4, take $t_{0}, R>0$ large enough so that

$$
\mathbb{P}\left(\mathcal{A}_{1}\left(t_{0}, R, \varepsilon\right)\right)>1-\varepsilon^{\prime} / 2
$$

and note that by Lemma 3.1, for the choice of $m$ made in (3.33), $\mathbb{P}\left(\mathcal{A}_{2}(m)\right)>1-\varepsilon^{\prime} / 2$.

Hence (3.24) holds and for $\omega \in \mathcal{A}_{1}\left(t_{0}, R, \varepsilon\right) \cap \mathcal{A}_{2}(m) \cap \Omega^{\prime}$, by (3.30) and (3.32),

$$
\begin{aligned}
\liminf _{t} & \frac{1}{t} \log E_{0}\left[\exp \left(-\int_{0}^{t} \widetilde{V}\left(Z_{s}, \omega\right) \mathrm{d} s\right), Z_{t} \in t B(v, r)\right] \\
> & -\widetilde{\alpha}_{\lambda}(v)+\lambda s_{1}-\varepsilon_{1} / 2 .
\end{aligned}
$$

To complete the proof, consider two cases according to the value of $\widetilde{\alpha}_{\lambda=0}^{\prime}(v)_{+}$.

Case $1: \widetilde{\alpha}_{\lambda=0}^{\prime}(v)_{+}<1$. Then $\widetilde{\alpha}_{\lambda}^{\prime}(v)_{+}<1$ for all $\lambda>0$. Hence, $I(v)=\widetilde{\alpha}_{0}(v)$.

For $\lambda>0$ sufficiently small, (3.34) leads to

$$
\liminf \frac{1}{t} \log E_{0}\left[\exp \left(-\int_{0}^{t} \widetilde{V}\left(Z_{s}, \omega\right) \mathrm{d} s\right), Z_{t} \in t B(v, r)\right] \geq-I(v)-3 \varepsilon_{1} / 4
$$

in contradiction with (3.31).

Case 2: $\widetilde{\alpha}_{\lambda=0}^{\prime}(v)_{+} \geq 1$. As in [37], let $\lambda_{\infty}(v):=\inf \left\{\lambda \in \mathbb{Q} ; \lambda>0\right.$ and $\left.\widetilde{\alpha}_{\lambda}^{\prime}(v)_{+}<1\right\}$. Then

$$
\lambda_{\infty}>0 \text { and } \widetilde{\alpha}_{\lambda_{\infty}}^{\prime}(v)_{+} \leq 1 \leq \widetilde{\alpha}_{\lambda_{\infty}}^{\prime}(v)_{-}
$$

since we assumed that $\widetilde{\alpha}_{\lambda=0}^{\prime}(v)_{+} \geq 1$ and by concavity of $\widetilde{\alpha} .(v)$.

If $\widetilde{\alpha}_{\lambda_{\infty}}^{\prime}(v)_{+}<1$, there are values of $s_{1}<s_{2}<1$ such that for $s_{1}<1$ sufficiently close to 1 , ]$s_{1}, s_{2}\left[\cap\left[\widetilde{\alpha}_{\lambda_{\infty}}^{\prime}(v)_{+}, \widetilde{\alpha}_{\lambda_{\infty}}^{\prime}(v)_{-}\right] \neq \emptyset\right.$ and $\lambda_{\infty} s_{1}>\lambda_{\infty}-\varepsilon_{1} / 5$. Then by $(3.34)$,

$$
\liminf \frac{1}{t} \log E_{0}\left[\exp \left(-\int_{0}^{t} \widetilde{V}\left(Z_{s}, \omega\right) \mathrm{d} s\right), Z_{t} \in t B(v, r)\right] \geq-I(v)-3 \varepsilon_{1} / 5
$$

in contradiction with (3.31). 
If $\widetilde{\alpha}_{\lambda_{\infty}}^{\prime}(v)_{+}=1$. Then $\widetilde{\alpha}_{\lambda}^{\prime}(v)_{+}<1$ for all $\lambda>\lambda_{\infty}$ and $\widetilde{\alpha}_{\lambda}^{\prime}(v)_{+} \uparrow 1$ as $\lambda \downarrow \lambda_{\infty}$. Therefore, there are values of $s_{1}<s_{2}<1$ and $\lambda>\lambda_{\infty}$ such that if $s_{1}$ is sufficiently close to 1 and $\lambda$ is sufficiently close to $\lambda_{\infty}$, then ]$s_{1}, s_{2}\left[\cap\left[\widetilde{\alpha}_{\lambda}^{\prime}(v)_{+}, \widetilde{\alpha}_{\lambda}^{\prime}(v)_{-}\right] \neq \emptyset\right.$ and by $(3.34)$,

$$
\liminf \frac{1}{t} \log E_{0}\left[\exp \left(-\int_{0}^{t} \widetilde{V}\left(Z_{s}, \omega\right) \mathrm{d} s\right), Z_{t} \in t B(v, r)\right] \geq-I(v)-3 \varepsilon_{1} / 5
$$

in contradiction with (3.31).

\subsection{Application to the Brownian motion with constant drift}

From the LDP for the speed of Brownian motion, Varadhan's lemma, one can obtain a LDP for Brownian motion in a random potential with a constant drift as in ([37], Thm. 4.7) by verifying the additional condition (2.1.9) of [12]. But since an upper gaussian estimate suffices, it is also verified for a stationary potential. This in turn leads to the observation of a transition from a sub-ballistic to a ballistic regime according to the strength of the drift. A similar phenomenon is proved for the random walk in a random potential in ([14], Thm. B(a)) and in ([25], Rem. 1.11).

For $h \in \mathbb{R}^{d}$, the quenched path measures of the Brownian motion in the random potential $V$ with drift $h$ is given by

$$
Q_{t, \omega}^{h}(A):=\frac{1}{S_{t, \omega}^{h}} E_{0}\left[\exp \left(h \cdot Z_{t}-\int_{0}^{t} V\left(Z_{s}\right) \mathrm{d} s\right), A\right], \quad t>0 .
$$

where $S_{t, \omega}^{h}=E_{0}\left[\exp \left(h \cdot Z_{t}-\int_{0}^{t} V\left(Z_{s}\right) \mathrm{d} s\right)\right]$.

The transition from a sub-ballistic to a ballistic regime appears clearly when described by the dual norm $\alpha_{\lambda}^{*}(y):=\sup _{x \neq 0} \frac{x \cdot y}{\alpha_{\lambda}(x)}$ for $y \in \mathbb{R}^{d}$ and $\lambda \geq-\underline{V}$.

Proposition 3.6. Let $V$ be a non-negative, stationary and ergodic potential which satisfies conditions (1.6), (1.7) and (1.8) of Theorem 1.1.

Then for $h \in \mathbb{R}^{d}$,

$$
\alpha_{-\underline{V}}^{*}(h) \leq 1 \quad \text { if and only if } \quad \lim _{t} \frac{1}{t} \log S_{t, \omega}^{h}=0 \text { a.s. }
$$

Moreover, when $\alpha_{-\underline{V}}^{*}(h)>1, \lim _{t} \frac{1}{t} \log S_{t, \omega}^{h}=\lambda_{h}>0$ where $\lambda=\lambda_{h}$ is the unique solution of $\alpha_{\lambda}^{*}(h)=1$.

The proof of ([37], Thm. 5.4.7) (see also [14], Sect. 5) holds with minor modifications. In particular, note that to justify that $\alpha_{\lambda}^{*}(h) \rightarrow 0$ as $\lambda \rightarrow \infty$, the inequality $\left.(2.8), \alpha_{\lambda}(x) \geq \sqrt{2(\lambda+\underline{V}}\right)|x|$, suffices.

\section{EXAMPLES}

In this section, we present some examples of potentials which satisfy the sufficient conditions of Theorem 1.1.

\subsection{A Poissonian potential: Lacoin's potential}

In this section, we show that the potentials introduced by Lacoin in [21], [22] satisfy the conditions of Theorem 1.1.

Their interest stems from the fact that the relations satisfied by their scaling exponents differ substantially from those established by Wüthrich [39-41] for a potential of the form (1.11) where $W$ has compact support.

These potentials are constructed from a Poisson Boolean model. Let $\Omega:=\left\{\omega=\left(\omega_{i}, r_{i}\right)_{i \geq 1}, \omega_{i} \in \mathbb{R}^{d}, r_{i} \geq 1\right\}$ be a Poisson point process in $\mathbb{R}^{d} \times[1, \infty[, d \geq 1$, whose intensity measure is given by Leb $\times \nu$ where $\nu$ is a 
probability measure on $[1, \infty[$ which depends on a parameter $\delta>0$ and is defined by

$$
\nu\left(\left[r, \infty[)=r^{-\delta}, \quad r \geq 1 .\right.\right.
$$

Note that each Poisson cloud $\omega \in \Omega$ is a locally finite subset of $\mathbb{R}^{d} \times\left[1, \infty\left[\right.\right.$. Index $\left(\omega_{i}, r_{i}\right)$ so that $\left(\left|\omega_{i}\right|, i \geq 1\right)$ is an increasing sequence. See ([24], Sect. 1.4) for an alternative description of this model and [19] for results on the percolative properties of the balls $B\left(\omega_{i}, r_{i}\right)$.

Given $\gamma>0$, Lacoin's potential $V: \mathbb{R}^{d} \times \Omega \longrightarrow[0, \infty[$ is defined by

$$
V(x, \omega):=\sum_{i=1}^{\infty} r_{i}^{-\gamma} \mathbf{1}_{B\left(\omega_{i}, r_{i}\right)}(x) .
$$

The behavior of this model depends on the positive parameters $\delta$ and $\gamma$. For $\gamma+\delta>d$, the potential is finite a.s. and the survival functions are strictly positive. Additional basic properties of this potential are gathered in the following lemma taken from [23]. The volume of the unit ball in $\mathbb{R}^{d}$ is denoted by $\mathcal{L}_{d}$.

Proposition 4.1. $\mathbb{P}$ - a.s. $V(x, \omega)$ is finite for every $x \in \mathbb{R}^{d}$ if and only if $\gamma+\delta-d>0$. In this case,

$$
\mathbb{E}[V(0)]=\frac{\mathcal{L}_{d} \delta}{\gamma+\delta-d}, \quad \operatorname{Var}[V(0)]=\frac{\mathcal{L}_{d} \delta}{2 \gamma+\delta-d}
$$

and for all $R>0, s \in \mathbb{R}$,

$$
\mathbb{E}\left[\exp \left(s \sum_{i=1}^{\infty} r_{i}^{-\gamma} \mathbf{1}_{\left\{\left|\omega_{i}\right| \leq r_{i}+R\right\}}\right)\right]=\exp \left(\int_{1}^{\infty} \delta \mathcal{L}_{d}(r+R)^{d} r^{-\delta-1}\left(e^{s r^{-\gamma}}-1\right) d r\right)
$$

is finite and there are positive constants $c_{7}(d, \delta, \gamma), c_{8}(d, \delta, \gamma)$ such that for all $x \in \mathbb{R}^{d},|x|>1$,

$$
c_{7}|x|^{d-\delta-2 \gamma} \leq \mathbb{C o v}(V(0), V(x)) \leq c_{8}|x|^{d-\delta-2 \gamma} .
$$

Moreover, the potential is ergodic.

In order to show that Lacoin's potential satisfies the conditions of Theorem 1.1, we first prove a weak independence property similar to ([18], Lem. 6). The method previously used in ([23], Lem. 2.6) lead to a weaker result.

Lemma 4.2. Assume that $\gamma+\delta-d>0$.

Then there is a constant $C=C(\gamma, \delta, d)$ such that for all $\varepsilon>0$, for all $R_{0}>1$ and $R>C \varepsilon^{-1 /(\gamma+\delta-d)} \vee 2 R_{0}$,

$$
\mathbb{P}\left(\sup _{y \in B\left(0, R_{0}\right)} \sum_{\omega_{j} \notin B(0, R)} r_{j}^{-\gamma} \mathbf{1}_{B\left(\omega_{j}, r_{j}\right)}(y)>\varepsilon\right) \leq \exp \left(-2 \varepsilon R^{\gamma}\right) .
$$

Proof. Let $R>2 R_{0}>R_{0}>1$. Then for all $y \in B\left(0, R_{0}\right)$ and $\omega_{j} \notin B(0, R)$,

$$
\left|\omega_{j}\right|<\left|\omega_{j}-y\right|+|y|<\left|\omega_{j}-y\right|+R_{0}<2\left|\omega_{j}-y\right|
$$

Hence

$$
\sup _{y \in B\left(0, R_{0}\right)} \sum_{\omega_{j} \notin B(0, R)} r_{j}^{-\gamma} \mathbf{1}_{B\left(\omega_{j}, r_{j}\right)}(y) \leq \sum_{\omega_{j} \notin B(0, R)} r_{j}^{-\gamma} \mathbf{1}_{\left\{\left|\omega_{j}\right|<2 r_{j}\right\}} .
$$


Moreover, by Campbell's theorem, for all $s>0$,

$$
\begin{aligned}
\log \mathbb{E}\left[\exp \left(s \sum_{\omega_{j} \notin B(0, R)} r_{j}^{-\gamma} \mathbf{1}_{\left\{\left|\omega_{j}\right|<2 r_{j}\right\}}\right)\right] & =\int_{1}^{\infty} \int_{\mathbb{R}^{d}}\left[\exp \left(s r^{-\gamma} \mathbf{1}_{\{R<|z|<2 r\}}\right)-1\right] d z \delta r^{-\delta-1} d r \\
& =\delta \mathcal{L}_{d} \int_{R / 2}^{\infty}\left((2 r)^{d}-R^{d}\right)\left(\exp \left(s r^{-\gamma}\right)-1\right) r^{-\delta-1} d r \\
& \leq C s \exp \left(s 2^{\gamma} R^{-\gamma}\right) \int_{R / 2}^{\infty} r^{d-\gamma-\delta-1} d r \\
& \leq C s \exp \left(s 2^{\gamma} R^{-\gamma}\right) R^{d-\delta-\gamma} .
\end{aligned}
$$

Then by Markov's inequality, (4.5) and (4.6) with $s=4 R^{\gamma}$, there exists $C=C(d, \delta, \gamma)$ such that for all $\varepsilon>0$, for all $R_{0}>1, R>\left(\frac{C}{2 \varepsilon}\right)^{\frac{1}{\gamma+\delta-d}} \vee\left(2 R_{0}\right)$,

$$
\mathbb{P}\left[\sup _{y \in B\left(0, R_{0}\right)} \sum_{\omega_{j} \notin B(0, R)} r_{j}^{-\gamma} \mathbf{1}_{B\left(\omega_{j}, r_{j}\right)}(y)>\varepsilon\right] \leq \exp \left(C R^{d-\delta}-4 \varepsilon R^{\gamma}\right)<\exp \left(-2 \varepsilon R^{\gamma}\right) .
$$

We are now ready to verify the hypothesis of Theorem 1.1 .

Proposition 4.3. Assume that $\gamma+\delta-d>0$. Conditions (1.6), (1.7) and (1.8) are satisfied, the Lyapunov exponents $\alpha(\cdot)$ is a norm and

$$
\lambda_{V}=\underline{V}=0
$$

Proof. To check that all moments of $\sup _{x \in B(0)} V(x, \cdot)$ are finite, note that

$$
\sup _{B(0)} V(\cdot, \omega) \leq \sum_{i=1}^{\infty} r_{i}^{-\gamma} \mathbf{1}_{\left\{\left|\omega_{i}\right| \leq r_{i}+1\right\}}
$$

and by (4.2),

$$
\mathbb{E}\left[\exp \left(\sum_{i=1}^{\infty} r_{i}^{-\gamma} \mathbf{1}_{\left\{\left|\omega_{i}\right| \leq r_{i}+1\right\}}\right)\right]=\exp \left(\int_{1}^{\infty} \delta \mathcal{L}_{d}(r+1)^{d} r^{-\delta-1}\left(e^{r^{-\gamma}}-1\right) d r\right)<\infty
$$

(since $e^{r^{-\gamma}}-1<2 r^{-\gamma}$ when $r$ is large enough and $\gamma+\delta-d>0$ ).

For $\mathbb{P}$-almost all Poisson clouds $\omega=\left(\omega_{i}, r_{i}\right)_{i \geq 1},\left(\omega_{i}, r_{i}\right) \in \mathbb{R}^{d} \times[1,2]$ infinitely often. Then set $u(\omega)=\omega_{j}$ where $\left(\omega_{j}, r_{j}\right)$ is the point of $\omega$ in the set $\mathbb{R}^{d} \times[1,2]$ with $\left|\omega_{j}\right|$ minimum.

Choose $\epsilon=2^{-\gamma}$ and $\rho=1$, then for all $x \in B(u, \rho)$, we have that

$$
V(x, \omega)=\sum_{k=1}^{\infty} r_{k}^{-\gamma} \mathbf{1}_{B\left(\omega_{k}, r_{k}\right)}(x) \geq r_{i}^{-\gamma} \mathbf{1}_{B\left(\omega_{i}, r_{i}\right)}(x)>2^{-\gamma} .
$$

Note that $(\operatorname{Leb} \times \nu)(B(0, t) \times[1,2])=\int_{|y|<t} \int_{1}^{2} \delta r^{-\delta-1} \mathrm{~d} y d r=\left(1-2^{-\delta}\right) \mathcal{L}_{d} t^{d}$. Then

$$
\mathbb{P}(|u|>t)=\mathbb{P}(\text { no points of the Poisson cloud are in } B(0, t) \times[1,2])=e^{-\left(1-2^{-\delta}\right) \mathcal{L}_{d} t^{d}} .
$$

Therefore $\mathbb{E}\left(|u|^{d}\right)<\infty$. Conditions (1.6) and (1.8) are satisfied. 
Then by Theorem 2.1, the Lyapunov exponents $\alpha(x)$ exist. Moreover, $\alpha$ is a norm. Indeed,

$$
V(x, \omega)=\sum_{i=1}^{\infty} r_{i}^{-\gamma} \mathbf{1}_{B\left(\omega_{i}, r_{i}\right)}(x) \geq \sum_{i ;\left(\omega_{i}, r_{i}\right) \in \mathbb{R}^{d} \times[1,2]} 2^{-\gamma} \mathbf{1}_{B\left(\omega_{i}, 1\right)}(x)
$$

which is a Poissonian potential constructed from a non-negative bounded measurable function with compact support. Then by ([37], Thm. 5.2.5), the associated Lyapunov exponents $\alpha^{1}(x)$ is a norm. And since $\alpha(x) \geq$ $\alpha^{1}(x), \alpha$ is also a norm.

To verify (1.7), we use Lemma 4.2 .

We now prove (4.7). For $x \in \mathbb{R}^{d}$ and $R>1$, write $V(x)=V_{1}(x)+V_{2}(x)$ where

$$
V_{1}(x)=\sum_{\omega_{i} \in B(0,2 R)} r_{i}^{-\gamma} \mathbf{1}_{B\left(\omega_{i}, r_{i}\right)}(x) \text { and } V_{2}(x)=\sum_{\omega_{i} \notin B(0,2 R)} r_{i}^{-\gamma} \mathbf{1}_{B\left(\omega_{i}, r_{i}\right)}(x) .
$$

For $\varepsilon>0$, let $R$ be large enough so that (4.4) holds. Then by the independence property of the Poisson point process,

$$
\begin{aligned}
\mathbb{P}\left(\sup _{x \in B(0, R)} V(x)<\varepsilon\right) & >\mathbb{P}\left(\sup _{x \in B(0, R)} V_{1}(x)<\varepsilon / 2, \sup _{x \in B(0, R)} V_{2}(x)<\varepsilon / 2\right) \\
& =\mathbb{P}\left(\sup _{x \in B(0, R)} V_{1}(x)<\varepsilon / 2\right) \mathbb{P}\left(\sup _{x \in B(0, R)} V_{2}(x)<\varepsilon / 2\right) \\
& >\exp \left(-C R^{d}\right)\left(1-\exp \left(-\varepsilon R^{\gamma}\right)\right) .
\end{aligned}
$$

The last inequality follows from Lemma 4.2 and the fact that $V_{1}(x)=0$ if no points of the Poisson cloud are in $B(0,2 R) \times[1, \infty[$. Hence, for all $\varepsilon>0$ and for all $R$ large enough,

$$
\mathbb{P}\left(\sup _{x \in B(0, R)} V(x)<\varepsilon\right)>0 .
$$

Let $\left(\varepsilon_{\ell} ; \ell \in \mathbb{N}\right)$ be a sequence of positive numbers such that $\varepsilon_{\ell} \rightarrow 0$ as $\ell \rightarrow \infty$. Then there is a sequence $R_{\ell} \rightarrow \infty$ such that for all $\ell \in \mathbb{N}, \mathbb{P}\left(\sup _{x \in B\left(0, R_{\ell}\right)} V(x)<\varepsilon_{\ell}\right)>0$.

By ergodicity, $\mathbb{P}$ - a.s. for each $\ell$ there is $z_{\ell}=z_{\ell}(\omega) \in \mathbb{R}^{d}$ such that $\sup _{x \in B\left(z_{\ell}, R_{\ell}\right)} V(x)<\varepsilon_{\ell}$.

Then $\underline{V}=0$ and (see [37], Sect. 3.1), $\mathbb{P}-$ a.s. for all $\ell \in \mathbb{N}$,

$$
\begin{aligned}
\lambda_{V} & \leq \lambda_{V}\left(B\left(z_{\ell}, R_{\ell}\right)\right) \\
& =\inf \left\{\int_{B\left(z_{\ell}, R_{\ell}\right)}\left[\frac{1}{2}|\nabla u|^{2}+V u^{2}\right] \mathrm{d} x, \int_{B\left(z_{\ell}, R_{\ell}\right)} u^{2} \mathrm{~d} x=1\right\} \\
& \leq C R_{\ell}^{-2}+\varepsilon_{\ell} .
\end{aligned}
$$

Let $\ell \rightarrow \infty$ to conclude.

\subsection{A Poissonian potential with polynomial tail}

Proposition 4.4. Let $V$ be a potential of the form

$$
V(x, \omega)=\sum_{j} W\left(x-\omega_{j}\right)
$$

where $\omega=\left(\omega_{j}\right)$ is a Poisson point process on $\mathbb{R}^{d}, d \geq 1$ with intensity given by Lebesgue measure and $W: \mathbb{R}^{d} \rightarrow$ $\left[0, \infty\left[\right.\right.$ is a measurable function, not negligible and which satisfies for $\gamma>d$ and for some positive constant $c_{9}$,

$$
W(x) \leq c_{9}\left(|x|^{-\gamma} \wedge 1\right) \text { for all } x \in \mathbb{R}^{d} .
$$


Then conditions (1.6), (1.7) and (1.8) are satisfied and the Lyapunov exponents $\alpha(\cdot)$ is a norm.

For $\gamma>d$, the survival functions are strictly positive. Precise estimates of the asymptotic behavior of the annealed survival function were obtained by Donsker and Varadhan for $\gamma>d+2$ and by Pastur [27] and Fukushima [18] for $d<\gamma<d+2$. The case where $\gamma=d+2$ is considered by Ôkura [26] and Chen and Kulik $[7,8]$ worked on the case $\gamma \leq d$.

The potential is ergodic since it is constructed from a Poisson point process (see for instance [24], Prop. 2.6).

Proof. Note that $\mathbb{P}-$ a.s. $\sup _{x \in[-1,1]^{d}} V(x, \cdot) \leq c_{9} \sum_{j} \widetilde{W}\left(\omega_{j}\right)$ where

$$
\widetilde{W}(x):=\mathbf{1}_{(|x| \leq 1+\sqrt{d})}+(1+\sqrt{d})^{\gamma}|x|^{-\gamma} \mathbf{1}_{(|x|>1+\sqrt{d})}, \quad x \in \mathbb{R}^{d} .
$$

Hence for $\theta>0$,

$$
\int_{\mathbb{R}^{d}}(\exp (\theta \widetilde{W}(x))-1) \mathrm{d} x<\int_{|x| \leq 1+\sqrt{d}}\left(e^{\theta}-1\right) \mathrm{d} x+e^{\theta} \int_{|x|>1+\sqrt{d}}(1+\sqrt{d})^{\gamma}|x|^{-\gamma} \mathrm{d} x<\infty .
$$

Then by Campbell's theorem for all $\theta \in \mathbb{R}, \mathbb{E}\left[\exp \left(\theta \sup _{x \in[-1,1]^{d}} V(x)\right)\right]<\infty$. This condition also appears as Assumption 2 in [17]. In particular, $\sup _{x \in[-1,1]^{d}} V$ has finite moments of all order and condition (1.6) holds.

Since $W$ is not negligible, there are $\varepsilon>0$ and $\rho>0$ such that

$$
\operatorname{Leb}(\{W>\varepsilon\} \cap B(0, \rho))>\varepsilon .
$$

Then condition (1.8) of Theorem 2.2 is satisfied with $u(\omega):=\omega_{1}$ where $\omega=\left(\omega_{i}\right)_{i \geq 1}$ is an enumeration of the points of the Poisson cloud so that $\left|\omega_{i}\right| \leq\left|\omega_{i+1}\right|, i \geq 1$. Indeed,

$$
\begin{aligned}
\mathbb{E}\left(\left|\omega_{1}\right|^{d}\right) & =d \int_{0}^{\infty} t^{d-1} \mathbb{P}\left(\left|\omega_{1}\right|>t\right) \mathrm{d} t \\
& =d \int_{0}^{\infty} t^{d-1} \mathbb{P}(\text { no points of the Poisson cloud are in } B(0, t)) \mathrm{d} t \\
& =d \int_{0}^{\infty} t^{d-1} \exp \left(-\mathcal{L}_{d} t^{d}\right) \mathrm{d} t=d / \mathcal{L}_{d}<\infty
\end{aligned}
$$

And $\operatorname{Leb}(\{V(\cdot, \omega)>\varepsilon\} \cap B(u, \rho)) \geq \operatorname{Leb}\left(\left\{W\left(\cdot-\omega_{1}\right)>\varepsilon\right\} \cap B\left(\omega_{1}, \rho\right)\right)>\varepsilon$.

Therefore, for $\gamma>d$, by Theorem 2.2 , there is a non-random semi-norm $\alpha(\cdot)$ on $\mathbb{R}^{d}$, such that

$$
\lim _{|x| \rightarrow \infty} \frac{1}{|x|}|a(0, x, \omega)-\alpha(x)|=0 \quad \mathbb{P} \text { - a.s. }
$$

Then by comparison with a Poissonian potential constructed from $W$ with compact support, the general argument given in ([37], pp. 234-236) shows that $\alpha$ is actually a norm.

To verify that $\lambda_{V}=\underline{V}=0$, it is possible to proceed as in Section 4.1 with the appropriate version of Lemma 4.2 given below.

Lemma 4.5. Assume that $\gamma>d$. Then there is a positive constant $C=C(\gamma, d)$ such that for all $\varepsilon>0$, for all $r>1$ and $R>C \varepsilon^{-1 /(\gamma-d)} \vee 2 r$,

$$
\mathbb{P}\left(\sup _{y \in B(0, r)} \sum_{\omega_{j} \notin B(0, R)}\left|y-\omega_{j}\right|^{-\gamma}>\varepsilon\right) \leq \exp \left(-4 \varepsilon R^{\gamma}\right) .
$$


Remark. Note that, by Campbell's theorem, for $|x|>2$,

$$
\begin{aligned}
\operatorname{Cov}(V(0), V(x)) & =\int_{\mathbb{R}^{d}} W(u) W(x-u) d u \\
& \leq C \int_{\mathbb{R}^{d}}\left(|u|^{-\gamma} \wedge 1\right)\left(|x-u|^{-\gamma} \wedge 1\right) d u \\
& \leq C \int_{B(0)}|x-u|^{-\gamma} d u+C \int_{B(0)^{c}}|u|^{-\gamma}|x-u|^{-\gamma} \mathbf{1}_{\{|u|<|x-u|\}} d u \\
& <C|x|^{-\gamma}+C \int_{B(0)^{c}}|u|^{-\gamma}\left(\frac{|x|}{2}\right)^{-\gamma} \mathbf{1}_{\{|u|<|x-u|\}} d u \\
& <C|x|^{-\gamma} .
\end{aligned}
$$

Moreover, if there is a positive constant $c_{10}$ such that $W(x) \geq c_{10}\left(|x|^{-\gamma} \wedge 1\right)$ for all $x \in \mathbb{R}^{d}$ then $\operatorname{Cov}(V(0), V(x))>C \int_{B(0)}|x-u|^{-\gamma} d u>C|x|^{-\gamma}$.

\subsection{Ruess' potential}

Ruess [33] gave an example of a two-dimensional Brownian motion in a stationary potential constructed from a planar Poissonian tessellation. A line in the plane is parametrized by its distance to the origin, denoted by $\rho$, and the angle $\theta \in[0, \pi[$ formed by the line and the horizontal axis. Take $\rho \in \mathbb{R}$ with the convention that $\rho>0$ if the line intersects the horizontal axis on the positive side and $\rho \leq 0$ otherwise. Then consider a Poisson point process on $\mathbb{R} \times[0, \pi[$ with intensity measure given by $\nu$ Leb $\times \mu$ where $\nu>0$ and $\mu$ is the uniform measure on $[0, \pi[$.

Fix $m, M \in[0, \infty[, m<M$ and $R \in] 0, \infty$. Then the potential $V(x, \omega)=m$ if $x$ is at a distance less than $R$ of one of the lines of the environment and $V(x, \omega)=M$ otherwise.

For these potentials, conditions (1.6) and (1.8) are satisfied. It is also clear from (1.4) that $\lambda_{V}=m$. Hence, $\lambda_{V}=m=\underline{V}$. Therefore the shape Theorem 2.2 and the LDP given in Theorem 1.1 hold for this family of potentials. The regularity of the potential, as defined in [33], is not needed for these results. However, for regularized versions of the potentials, [33] gave a variational expression for quenched free energy.

\section{REFERENCES}

[1] S.N. Armstrong and H.V. Tran, Stochastic homogenization of viscous Hamilton-Jacobi equations and applications. Anal. Partial Differ. Equ. 7 (2014) 1969-2007.

[2] M. Björklund, The asymptotic shape theorem for generalized first passage percolation. Ann. Probab. 38 (2010) $632-660$.

[3] D. Boivin and Y. Derriennic, The ergodic theorem for additive cocycles of $\mathbb{Z}^{d}$ or $\mathbb{R}^{d}$. Ergodic Theory Dyn. Syst. 11 (1991) 19-39.

[4] D. Boivin, First passage percolation: the stationary case. Probab. Theory Related Fields 86 (1990) 491-499.

[5] A. Braides and A. Piatnitski, Homogenization of surface and length energies for spin systems. J. Funct. Anal. 264 (2013) 1296-1328.

[6] M. Broise, Y. Déniel and Y. Derriennic, Réarrangement, inégalités maximales et théorèmes ergodiques fractionnaires. Ann. Inst. Fourier (Grenoble) 39 (1989) 689-714.

[7] X. Chen, Quenched asymptotics for Brownian motion of renormalized Poisson potential and for the related parabolic Anderson models. Ann. Probab. 40 (2012) 1436-1482.

[8] X. Chen and A.M. Kulik, Brownian motion and parabolic Anderson model in a renormalized Poisson potential. Ann. Inst. Henri Poincaré Probab. Stat. 48 (2012) 631-660.

[9] K.L. Chung and Z. Zhao, From Brownian motion to Schroödinger's equation. Springer- Verlag, Berlin (1995).

[10] J.T. Cox and R. Durrett, Some limit theorems for percolation processes with necessary and sufficient conditions. Ann. Probab. (1981) 583-603.

[11] E.B. Davies, Heat kernels and spectral theory. Cambridge University Press, Cambridge (1989).

[12] J.-D. Deuschel and D.W. Stroock, Large deviations. Academic Press, Inc., Boston, MA (1989).

[13] M.D. Donsker and S.R.S. Varadhan, Asymptotic evaluation of certain Wiener integrals for large time, in Functional integration and its applications (Proc. Internat. Conf., London, 1974) (1975) 15-33. 
[14] M. Flury, Large deviations and phase transition for random walks in random nonnegative potentials. Stochastic Process. Appl. 117 (2007) 596-612.

[15] M. Flury, A note on the ballistic limit of random motion in a random potential. Electr. Commun. Probab. 13 (2008) 393-400.

[16] R. Fukushima, Asymptotics for the Wiener sausage among Poissonian obstacles. J. Stat. Phys. 133 (2008) 639-657.

[17] R. Fukushima, From the Lifshitz tail to the quenched survival asymptotics in the trapping problem. Electron. Commun. Probab. 14 (2009) 435-446.

[18] R. Fukushima, Second order asymptotics for Brownian motion in a heavy tailed Poissonian potential. Markov Process. Related Fields 17 (2011) 447-482.

[19] J.-B. Gouéré, Subcritical regimes in the Poisson Boolean model of continuum percolation. Ann. Probab. 36 (2008) 1209-1220.

[20] U. Krengel, Ergodic theorems. Walter de Gruyter \& Co., Berlin (1985).

[21] H. Lacoin, Superdiffusivity for Brownian motion in a Poissonian potential with long range correlation: I: Lower bound on the volume exponent. Ann. Inst. Henri Poincaré Probab. Stat. 48 (2012) 1010-1028.

[22] H. Lacoin, Superdiffusivity for Brownian motion in a Poissonian potential with long range correlation II: Upper bound on the volume exponent. Ann. Inst. Henri Poincaré Probab. Stat. 48 (2012) 1029-1048.

[23] T.T.H. Lê, Exposants de Lyapunov et potentiel aléatoire. Université de Bretagne Occidentale, France (2015).

[24] R. Meester and R. Roy, Continuum percolation. Cambridge University Press, Cambridge (1996).

[25] J.-C. Mourrat, Lyapunov exponents, shape theorems and large deviations for the random walk in random potential. $A L E A$ Lat. Am. J. Probab. Math. Stat. 9 (2012) 165-209.

[26] H. Ôkura, An asymptotic property of a certain Brownian motion expectation for large time. Proc. Jpn. Acad. Ser. A Math. Sci. 57 (1981) 155-159.

[27] L.A. Pastur, The behavior of certain Wiener integrals as $t \rightarrow \infty$ and the density of states of Schrödinger equations with random potential. Teoret. Mat. Fiz. 32 (1977) 88-95.

[28] F. Rassoul-Agha, Large deviations for random walks in a mixing random environment and other (non-Markov) random walks. Commun. Pure Appl. Math. 57 (2004) 1178-1196.

[29] F. Rassoul-Agha and T. Seppäläinen, Process-level quenched large deviations for random walk in random environment. Ann. Inst. Henri Poincaré Probab. Stat. 47 (2011) 214-242.

[30] F. Rassoul-Agha and T. Seppäläinen, Quenched point-to-point free energy for random walks in random potentials. Probab. Theory Related Fields 158 (2014) 711-750.

[31] F. Rassoul-Agha, T. Seppäläinen and A. Yilmaz, Quenched free energy and large deviations for random walks in random potentials. Comm. Pure Appl. Math. 66 (2013) 202-244.

[32] J.M. Rosenbluth, Quenched large deviation for multidimensional random walk in random environment: A variational formula. Ph.D. thesis, New York University (2006).

[33] J. Rueß, A variational formula for the Lyapunov exponent of Brownian motion in stationary ergodic potential. ALEA Lat. Am. J. Probab. Math. Stat. 11 (2014) 679-709.

[34] J. Rueß, Continuity results and estimates for the Lyapunov exponent of Brownian motion in stationary potential. Braz. J. Probab. Stat. 30 (2016) 435-463.

[35] C. Schroeder, Green's functions for the Schrödinger operator with periodic potential. J. Funct. Anal. 77 (1988) $60-87$.

[36] A.-S. Sznitman, Shape theorem, Lyapounov exponents, and large deviations for Brownian motion in a Poissonian potential. Comm. Pure Appl. Math. 47 (1994) 1655-1688.

[37] A.-S. Sznitman, Brownian motion, obstacles and random media. Springer-Verlag, Berlin (1998).

[38] S.R.S. Varadhan, Large deviations for random walks in a random environment. Dedicated to the memory of Jürgen K. Moser. Commun. Pure Appl. Math. 56 (2003) 1222-1245.

[39] M.V. Wüthrich, Scaling identity for crossing Brownian motion in a Poissonian potential. Probab. Theory Related Fields 112 (1998) 299-319.

[40] M.V. Wüthrich, Superdiffusive behavior of two-dimensional Brownian motion in a Poissonian potential. Ann. Probab. 26 (1998) 1000-1015.

[41] M.V. Wüthrich, Fluctuation results for Brownian motion in a Poissonian potential. Ann. Inst. Henri Poincaré Probab. Statist. 34 (1998) 279-308.

[42] A. Yilmaz, Quenched large deviations for random walk in a random environment. Commun. Pure Appl. Math. 62 (2009) $1033-1075$.

[43] A. Yilmaz, Equality of averaged and quenched large deviations for random walks in random environments in dimensions four and higher. Probab. Theory Related Fields 149 (2011) 463-491.

[44] A. Yilmaz and O. Zeitouni, Differing averaged and quenched large deviations for random walks in random environments in dimensions two and three. Commun. Math. Phys. 300 (2010) 243-271.

[45] M.P.W. Zerner, Directional decay of the Green's function for a random nonnegative potential on $\mathbf{Z}^{d}$. Ann. Appl. Probab. 8 (1998) 246-280 